\title{
Broadcasting Correlated Vector Gaussians
}

\author{
Lin Song, Jun Chen, Member, IEEE, and Chao Tian, Senior Member, IEEE
}

\begin{abstract}
The problem of sending two correlated vector Gaussian sources over a bandwidth-matched two-user scalar Gaussian broadcast channel is studied in this paper, where each receiver wishes to reconstruct its target source under a covariance distortion constraint. We derive a lower bound on the optimal tradeoff between the transmit power and the achievable reconstruction distortion pair. Our derivation is based on a new bounding technique which involves the introduction of appropriate remote sources. Furthermore, it is shown that this lower bound is achievable by a class of hybrid schemes for the special case, where the weak receiver wishes to reconstruct a scalar source under the mean squared error distortion constraint.
\end{abstract}

Index Terms-Broadcast channel, dirty paper coding, Gaussian source, joint source-channel coding, Wyner-Ziv coding.

\section{INTRODUCTION}

$\mathbf{U}$ NLIKE in point-to-point communication systems where the source-channel separation architecture is optimal [1], in multi-user systems, a separation-based architecture is usually suboptimal. In such scenarios, hybrid schemes have emerged as a promising approach to gain performance improvement over either pure digital schemes (separationbased schemes) or pure analog schemes, e.g., in [2] for bandwidth-mismatch Gaussian source broadcast (see also [3]-[5] for variants of this problem), and in [6] for sending a bivariate Gaussian source over a Gaussian multiple access channel. Recently, building upon the important work by Bross et al. [7] as well as [8] and [9], Tian et al. [10] showed that, for the problem of broadcasting a bivariate Gaussian source, hybrid schemes are not only able to provide such performance improvement, they can in fact be optimal.

In this paper, we consider the problem of sending two correlated vector Gaussian sources over a bandwidth-matched two-user scalar Gaussian broadcast channel, where each receiver wishes to reconstruct its target source under a covariance distortion constraint (see Fig. 1). This can be

Manuscript received March 21, 2012; revised July 2, 2014; accepted March 8, 2015. Date of publication March 6, 2015; date of current version April 17, 2015. L. Song and J. Chen were supported in part by the Early Researcher Award from the Province of Ontario and in part by the Natural Science and Engineering Research Council of Canada through the Discovery Grant. This paper was presented at the 2012 IEEE International Symposium on Information Theory.

L. Song was with the Department of Electrical and Computer Engineering, McMaster University, Hamilton, ON L8S 4L8, Canada. She is now with the Institute of Network Coding, The Chinese University of Hong Kong, Hong Kong (e-mail: linsong@inc.cuhk.edu.hk).

J. Chen is with the Department of Electrical and Computer Engineering, McMaster University, Hamilton, ON L8S 4L8, Canada (e-mail: junchen@ece.mcmaster.ca).

C. Tian was with AT\&T Labs-Research, Bedminster, NJ 07921 USA. $\mathrm{He}$ is now with the University of Tennessee, Knoxville, TN 37996 USA (e-mail: chao.tian@utk.edu).

Communicated by Y. Steinberg, Associate Editor at Large.

Digital Object Identifier 10.1109/TIT.2015.2412540

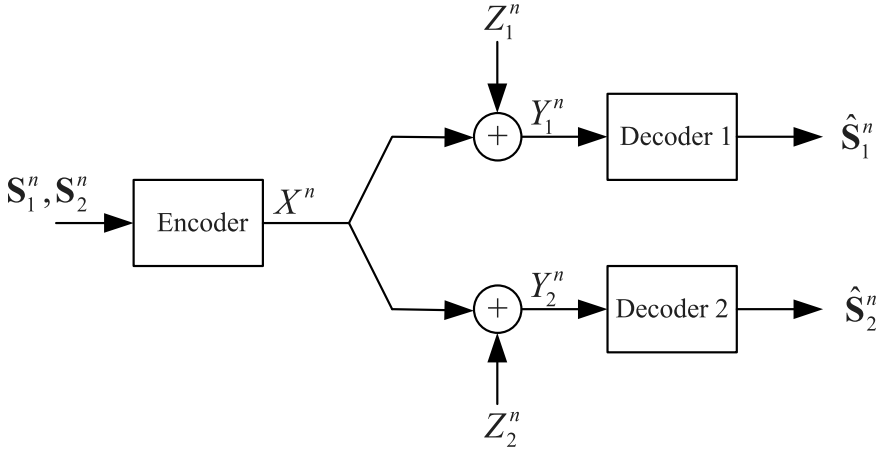

Fig. 1. Broadcasting correlated vector Gaussian sources.

viewed as a vector generalization of the problem studied in [7], [8], and [10]. We derive a lower bound on the optimal tradeoff between the transmit power and the achievable reconstruction distortion pair. Furthermore, it is shown that this lower bound is tight for the scenario, referred to as the vector-scalar case, where the weak receiver wishes to reconstruct a scalar source under the mean squared error distortion constraint. It is worth noting that the brute-force proof method in [7] and [10] is difficult to generalize to the problem being considered. Therefore, instead of seeking explicit upper and lower bounds and showing their tightness by direct comparison, we take a more conceptual approach in the present work. In particular, the derivation of our lower bound is based on a new bounding technique which involves the introduction of appropriate remote sources; moreover, to obtain a matching upper bound in the vector-scalar case, we construct a scheme with its parameters specified according to an optimization problem motivated by the lower bound. Another finding is that the optimal scheme is in general not unique. Indeed, we show that, in the vector-scalar case, the optimal tradeoff between the transmit power and the reconstruction distortion pair is achievable by a class of hybrid schemes, which includes the scheme proposed by Tian et al. [10] as an extremal example.

\section{Problem Definition}

Let $\mathbf{S}_{i}$ be an $m_{i} \times 1$ zero-mean random vector, $i=1,2$. We assume that $\mathbf{S}_{1}$ and $\mathbf{S}_{2}$ are jointly Gaussian with covariance matrix

$$
\Sigma_{\mathbf{S}_{1}, \mathbf{S}_{2}}=\left(\begin{array}{cc}
\Sigma_{\mathbf{S}_{1}} & \mathbb{E}\left[\mathbf{S}_{1} \mathbf{S}_{2}^{T}\right] \\
\mathbb{E}\left[\mathbf{S}_{2} \mathbf{S}_{1}^{T}\right] & \Sigma_{\mathbf{S}_{2}}
\end{array}\right)
$$

where $\Sigma_{\mathbf{S}_{i}}=\mathbb{E}\left[\mathbf{S}_{i} \mathbf{S}_{i}^{T}\right], i=1,2$. Let the broadcast channel additive noises $Z_{1}$ and $Z_{2}$ be two zero-mean Gaussian random variables, jointly independent of $\left(\mathbf{S}_{1}, \mathbf{S}_{2}\right)$, with variances $N_{1}$ and $N_{2}$, respectively; it is assumed that $N_{2}>N_{1}$. Let $\left\{\left(\mathbf{S}_{1}(t), \quad \mathbf{S}_{2}(t), \quad Z_{1}(t), \quad Z_{2}(t)\right)\right\}_{t=1}^{\infty}$ be i.i.d. copies of $\left(\mathbf{S}_{1}, \mathbf{S}_{2}, Z_{1}, Z_{2}\right)$. 
Definition 1: An $\left(n, P, \mathbf{D}_{1}, \mathbf{D}_{2}\right)$ source-channel broadcast code consists of an encoding function $f: \mathbb{R}^{m_{1} \times n} \times$ $\mathbb{R}^{m_{2} \times n} \rightarrow \mathbb{R}^{n}$ and two decoding functions $g_{i}: \mathbb{R}^{n} \rightarrow \mathbb{R}^{m_{i} \times n}$, $i=1,2$, such that

$$
\begin{aligned}
& \frac{1}{n} \mathbb{E}\left[X^{n}\left(X^{n}\right)^{T}\right] \leq P, \\
& \frac{1}{n} \mathbb{E}\left[\left(\mathbf{S}_{i}^{n}-\hat{\mathbf{S}}_{i}^{n}\right)\left(\mathbf{S}_{i}^{n}-\hat{\mathbf{S}}_{i}^{n}\right)^{T}\right] \preceq \mathbf{D}_{i}, \quad i=1,2,
\end{aligned}
$$

where $X^{n}=f\left(\mathbf{S}_{1}^{n}, \mathbf{S}_{2}^{n}\right)$ and $\hat{\mathbf{S}}_{i}^{n}=g_{i}\left(Y_{i}^{n}\right), i=1,2$, with $Y_{i}^{n}=X^{n}+Z_{i}^{n}, i=1,2$.

It is clear that the performance of any source-channel broadcast code depends on $\left(Z_{1}^{n}, Z_{2}^{n}\right)$ only through their marginal distributions. Therefore, we shall assume the broadcast channel is physically degraded and write $Z_{2}^{n}=Z_{1}^{n}+Z^{n}$, where $Z^{n}$ is a zero-mean Gaussian random vector with i.i.d. entries of variance $N_{2}-N_{1}$ and is independent of $Z_{1}^{n}$. It is also clear [11, Appendix 3.A] that there is no loss of optimality in assuming $\hat{\mathbf{S}}_{i}^{n}=g_{i}\left(Y_{i}^{n}\right)=\mathbb{E}\left[\mathbf{S}_{i}^{n} \mid Y_{i}^{n}\right], i=1,2$.

Definition 2: We say $\left(P, \mathbf{D}_{1}, \mathbf{D}_{2}\right)$ is achievable if there exists an $\left(n, P, \mathbf{D}_{1}, \mathbf{D}_{2}\right)$ source-channel broadcast code. Let $\mathcal{P D}$ denote the closure of the set of all achievable $\left(P, \mathbf{D}_{1}, \mathbf{D}_{2}\right)$.

Definition 3: Let $P\left(\mathbf{D}_{1}, \mathbf{D}_{2}\right)=\inf \left\{P:\left(P, \mathbf{D}_{1}, \mathbf{D}_{2}\right) \in \mathcal{P} \mathcal{D}\right\}$.

With the above definitions, it is clear that the fundamental problem in this joint source-channel coding scenario is to determine the function $P\left(\mathbf{D}_{1}, \mathbf{D}_{2}\right)$, which characterizes the optimal tradeoff between the transmit power and the achievable reconstruction distortion pair. ${ }^{1}$ Unless specified otherwise, we assume $\Sigma_{\mathbf{S}_{1}, \mathbf{S}_{2}} \succ \mathbf{0}$ and $\mathbf{D}_{i} \succ \mathbf{0}, i=1,2$.

The remainder of this paper is organized as follows. We derive a lower bound on $P\left(\mathbf{D}_{1}, \mathbf{D}_{2}\right)$ in Section III. It is shown in Section IV that, for the vector-scalar case, this lower bound is achievable by a class of hybrid schemes. We conclude the paper in Section V. Throughout this paper, the logarithm function is to base $e$.

\section{LOWER BOUND}

Let $\mathbf{U}_{i}$ be an $m_{i} \times 1$ zero-mean random vector, $i=1,2$. We assume that $\mathbf{U}_{1}$ and $\mathbf{U}_{2}$ are jointly Gaussian with covariance matrix

$$
\Sigma_{\mathbf{U}_{1}, \mathbf{U}_{2}}=\left(\begin{array}{cc}
\Sigma_{\mathbf{U}_{1}} & \mathbb{E}\left[\mathbf{U}_{1} \mathbf{U}_{2}^{T}\right] \\
\mathbb{E}\left[\mathbf{U}_{2} \mathbf{U}_{1}^{T}\right] & \Sigma_{\mathbf{U}_{2}}
\end{array}\right),
$$

where $\Sigma_{\mathbf{U}_{i}}=\mathbb{E}\left[\mathbf{U}_{i} \mathbf{U}_{i}^{T}\right], i=1,2$.

The main result of this section is the following theorem.

Theorem 1:

$$
\begin{aligned}
P\left(\mathbf{D}_{1}, \mathbf{D}_{2}\right) \geq & \inf _{\boldsymbol{\Theta}} \sup _{\Sigma_{\mathbf{U}_{1}, \mathbf{U}_{2}} \succ \mathbf{0}} N_{1} \frac{\left|\Sigma_{\mathbf{S}_{1}, \mathbf{S}_{2}}\right|\left|\boldsymbol{\Theta}_{2}+\Sigma_{\mathbf{U}_{2}}\right|}{|\boldsymbol{\Theta}|\left|\mathbf{D}_{2}+\Sigma_{\mathbf{U}_{2}}\right|} \\
& +\left(N_{2}-N_{1}\right) \frac{\left|\Sigma_{\mathbf{S}_{2}}+\Sigma_{\mathbf{U}_{2}}\right|}{\left|\mathbf{D}_{2}+\Sigma_{\mathbf{U}_{2}}\right|}-N_{2}
\end{aligned}
$$

with the infimum taken over $\left(m_{1}+m_{2}\right) \times\left(m_{1}+m_{2}\right)$ matrix $\boldsymbol{\Theta}$ subject to the constraints

$$
\begin{aligned}
& \mathbf{0} \prec \boldsymbol{\Theta} \preceq \Sigma_{\mathbf{S}_{1}, \mathbf{S}_{2}}, \\
& \mathbf{\Theta}_{1} \preceq \mathbf{D}_{1} .
\end{aligned}
$$

\footnotetext{
${ }^{1}$ This formulation is slightly different from that in [7] and [10], where the power $P$ is fixed, and the tradeoff between the reconstruction distortion pair is considered. We find the current formulation more suitable here, since both receivers are to reconstruct vector sources.
}

Here we assume that $\boldsymbol{\Theta}$ is partitioned to the form

$$
\boldsymbol{\Theta}=\left(\begin{array}{cc}
\Theta_{1} & \# \\
\# & \Theta_{2}
\end{array}\right),
$$

where $\boldsymbol{\Theta}_{i}$ is of size $m_{i} \times m_{i}$ for $i=1,2$.

Remark: It is interesting to note that the objective function on the right-hand side of (1) depends on $\Sigma_{\mathbf{U}_{1}, \mathbf{U}_{2}}$ only through $\Sigma_{\mathbf{U}_{2}}$. Therefore, one can simply take the supremum in (1) over $\Sigma_{\mathbf{U}_{2}} \succ \mathbf{0}$.

Remark: Theorem 1 is in fact closely related to [12, Th. 1]. A detailed explanation of the connections between these two results can be found in [13].

The following two elementary inequalities are needed for the proof of Theorem 1. For completeness, their proofs are given in Appendices A and B.

Lemma 1: For any $m \times n$ random matrices $\mathbf{W}$ and $\hat{\mathbf{W}}$,

$$
h(\mathbf{W} \mid \hat{\mathbf{W}}) \leq \frac{n}{2} \log \left|\frac{2 \pi e}{n} \mathbb{E}\left[(\mathbf{W}-\hat{\mathbf{W}})(\mathbf{W}-\hat{\mathbf{W}})^{T}\right]\right| .
$$

Lemma 2: Let $\mathbf{W}_{i}$ be an $m_{i} \times n$ zero-mean random matrix, $i=1$, 2. If $\frac{1}{n} \mathbb{E}\left[\left(\mathbf{W}_{1}^{T}, \mathbf{W}_{2}^{T}\right)^{T}\left(\mathbf{W}_{1}^{T}, \mathbf{W}_{2}^{T}\right)\right] \succ \mathbf{0}$, then

$$
h\left(\mathbf{W}_{1} \mid \mathbf{W}_{2}\right) \leq \frac{n}{2} \log \frac{\left|\frac{2 \pi e}{n} \mathbb{E}\left[\left(\mathbf{W}_{1}^{T}, \mathbf{W}_{2}^{T}\right)^{T}\left(\mathbf{W}_{1}^{T}, \mathbf{W}_{2}^{T}\right)\right]\right|}{\left|\frac{2 \pi e}{n} \mathbb{E}\left[\mathbf{W}_{2} \mathbf{W}_{2}^{T}\right]\right|}
$$

Now we proceed to prove Theorem 1.

Proof of Theorem 1: For any $\left(n, P, \mathbf{D}_{1}, \mathbf{D}_{2}\right)$ source-channel broadcast code, let $\hat{\mathbf{S}}_{i}^{n}=g_{i}\left(Y_{i}^{n}\right)=\mathbb{E}\left[\mathbf{S}_{i}^{n} \mid Y_{i}^{n}\right]$, $i=1,2$, and $\tilde{\mathbf{S}}_{2}^{n}=\mathbb{E}\left[\mathbf{S}_{2}^{n} \mid Y_{1}^{n}\right]$; furthermore, let

$$
\boldsymbol{\Theta}=\left(\begin{array}{cc}
\Theta_{1} & \Upsilon \\
\Upsilon^{T} & \boldsymbol{\Theta}_{2}
\end{array}\right)
$$

with $\boldsymbol{\Theta}_{1}=\frac{1}{n} \mathbb{E}\left[\left(\mathbf{S}_{1}^{n}-\hat{\mathbf{S}}_{1}^{n}\right)\left(\mathbf{S}_{1}^{n}-\hat{\mathbf{S}}_{1}^{n}\right)^{T}\right], \boldsymbol{\Theta}_{2}=\frac{1}{n} \mathbb{E}\left[\left(\mathbf{S}_{2}^{n}-\tilde{\mathbf{S}}_{2}^{n}\right)\right.$ $\left.\left(\mathbf{S}_{2}^{n}-\tilde{\mathbf{S}}_{2}^{n}\right)^{T}\right]$, and $\boldsymbol{\Upsilon}=\frac{1}{n} \mathbb{E}\left[\left(\mathbf{S}_{1}^{n}-\hat{\mathbf{S}}_{1}^{n}\right)\left(\mathbf{S}_{2}^{n}-\tilde{\mathbf{S}}_{2}^{n}\right)^{T}\right]$. Note that $\boldsymbol{\Theta}$ satisfies (2) and (3). Therefore, it suffices to show that

$$
\begin{aligned}
P \geq & N_{1} \frac{\left|\Sigma_{\mathbf{S}_{1}, \mathbf{S}_{2}}\right|\left|\boldsymbol{\Theta}_{2}+\Sigma_{\mathbf{U}_{2}}\right|}{|\boldsymbol{\Theta}|\left|\mathbf{D}_{2}+\Sigma_{\mathbf{U}_{2}}\right|} \\
& +\left(N_{2}-N_{1}\right) \frac{\left|\Sigma_{\mathbf{S}_{2}}+\Sigma_{\mathbf{U}_{2}}\right|}{\left|\mathbf{D}_{2}+\Sigma_{\mathbf{U}_{2}}\right|}-N_{2}
\end{aligned}
$$

for all $\Sigma_{\mathbf{U}_{1}, \mathbf{U}_{2}} \succ \mathbf{0}$.

Let $\left\{\mathbf{U}_{1}(t), \mathbf{U}_{2}(t)\right\}_{t=1}^{n}$ be i.i.d. copies of $\left(\mathbf{U}_{1}, \mathbf{U}_{2}\right)$. We assume that $\left(\mathbf{U}_{1}^{n}, \mathbf{U}_{2}^{n}\right)$ is independent of $\left(\mathbf{S}_{1}^{n}, \mathbf{S}_{2}^{n}, Z_{1}^{n}, Z^{n}\right)$. Define $\mathbf{V}_{i}^{n}=\mathbf{S}_{i}^{n}+\mathbf{U}_{i}^{n}, i=1,2$. Here $\mathbf{V}_{1}$ and $\mathbf{V}_{2}$ can be understood as the remote sources that should be reconstructed, yet the encoder only has access to $\mathbf{S}_{1}$ and $\mathbf{S}_{2}$. The introduction of $\left(\mathbf{V}_{1}, \mathbf{V}_{2}\right)$ is partly inspired by Ozarow's converse argument for the Gaussian multiple description problem [14] (see also [15]-[17]).

We shall first bound $I\left(\mathbf{V}_{2}^{n} ; Y_{2}^{n}\right)$. In view of the fact that

$$
0 \leq I\left(\mathbf{V}_{2}^{n} ; Y_{2}^{n}\right) \leq I\left(X^{n} ; Y_{2}^{n}\right) \leq \frac{n}{2} \log \frac{P+N_{2}}{N_{2}},
$$

we have

$$
I\left(\mathbf{V}_{2}^{n} ; Y_{2}^{n}\right)=\frac{n}{2} \log \frac{P+N_{2}}{\alpha P+N_{2}}
$$


for some $\alpha \in[0,1]$. On the other hand,

$$
\begin{aligned}
I\left(\mathbf{V}_{2}^{n} ; Y_{2}^{n}\right) & \\
= & h\left(\mathbf{V}_{2}^{n}\right)-h\left(\mathbf{V}_{2}^{n} \mid Y_{2}^{n}\right) \\
= & \frac{n}{2} \log \left|2 \pi e\left(\Sigma_{\mathbf{S}_{2}}+\Sigma_{\mathbf{U}_{2}}\right)\right|-h\left(\mathbf{V}_{2}^{n} \mid Y_{2}^{n}\right) \\
\geq & \frac{n}{2} \log \left|2 \pi e\left(\Sigma_{\mathbf{S}_{2}}+\Sigma_{\mathbf{U}_{2}}\right)\right|-h\left(\mathbf{V}_{2}^{n} \mid \hat{\mathbf{S}}_{2}^{n}\right) \\
\geq & \frac{n}{2} \log \left|\Sigma_{\mathbf{S}_{2}}+\Sigma_{\mathbf{U}_{2}}\right| \\
& -\frac{n}{2} \log \left|\frac{1}{n} \mathbb{E}\left[\left(\mathbf{V}_{2}^{n}-\hat{\mathbf{S}}_{2}^{n}\right)\left(\mathbf{V}_{2}^{n}-\hat{\mathbf{S}}_{2}^{n}\right)^{T}\right]\right| \\
\geq & \frac{n}{2} \log \left|\Sigma_{\mathbf{S}_{2}}+\Sigma_{\mathbf{U}_{2}}\right| \\
& -\frac{n}{2} \log \left|\frac{1}{n} \mathbb{E}\left[\left(\mathbf{S}_{2}^{n}-\hat{\mathbf{S}}_{2}^{n}\right)\left(\mathbf{S}_{2}^{n}-\hat{\mathbf{S}}_{2}^{n}\right)^{T}\right]+\Sigma_{\mathbf{U}_{2}}\right| \\
\geq & \frac{n}{2} \log \frac{\left|\Sigma_{\mathbf{S}_{2}}+\Sigma_{\mathbf{U}_{2}}\right|}{\left|\mathbf{D}_{2}+\Sigma_{\mathbf{U}_{2}}\right|},
\end{aligned}
$$

where (6) follows from Lemma 1. Combining (5) and (7) gives

$$
\frac{\left|\Sigma_{\mathbf{S}_{2}}+\Sigma_{\mathbf{U}_{2}}\right|}{\left|\mathbf{D}_{2}+\Sigma_{\mathbf{U}_{2}}\right|} \leq \frac{P+N_{2}}{\alpha P+N_{2}} .
$$

Now we proceed to bound $I\left(\mathbf{V}_{1}^{n} ; Y_{1}^{n} \mid \mathbf{V}_{2}^{n}\right)$. Since $h\left(Y_{2}^{n}\right) \leq \frac{n}{2} \log \left(2 \pi e\left(P+N_{2}\right)\right)$, it follows from (5) that

$$
h\left(Y_{2}^{n} \mid \mathbf{V}_{2}^{n}\right) \leq \frac{n}{2} \log \left(2 \pi e\left(\alpha P+N_{2}\right)\right) .
$$

By the entropy power inequality,

$$
\begin{aligned}
h\left(Y_{2}^{n} \mid \mathbf{V}_{2}^{n}\right) & \geq \frac{n}{2} \log \left(e^{\frac{2}{n} h\left(Y_{1}^{n} \mid \mathbf{V}_{2}^{n}\right)}+e^{\frac{2}{n} h\left(Z^{n}\right)}\right) \\
& =\frac{n}{2} \log \left(e^{\frac{2}{n} h\left(Y_{1}^{n} \mid \mathbf{V}_{2}^{n}\right)}+2 \pi e\left(N_{2}-N_{1}\right)\right),
\end{aligned}
$$

which, together with (9), implies

$$
h\left(Y_{1}^{n} \mid \mathbf{V}_{2}^{n}\right) \leq \frac{n}{2} \log \left(2 \pi e\left(\alpha P+N_{1}\right)\right) .
$$

Note that

$$
\begin{aligned}
& I\left(\mathbf{V}_{1}^{n} ; Y_{1}^{n} \mid \mathbf{V}_{2}^{n}\right) \\
& =h\left(Y_{1}^{n} \mid \mathbf{V}_{2}^{n}\right)-h\left(Y_{1}^{n} \mid \mathbf{V}_{1}^{n}, \mathbf{V}_{2}^{n}\right) \\
& \leq \frac{n}{2} \log \left(2 \pi e\left(\alpha P+N_{1}\right)\right)-h\left(Y_{1}^{n} \mid \mathbf{V}_{1}^{n}, \mathbf{V}_{2}^{n}\right) \\
& =\frac{n}{2} \log \frac{\alpha P+N_{1}}{N_{1}}-h\left(Y_{1}^{n} \mid \mathbf{V}_{1}^{n}, \mathbf{V}_{2}^{n}\right)+h\left(Y_{1}^{n} \mid \mathbf{S}_{1}^{n}, \mathbf{S}_{2}^{n}\right) \\
& =\frac{n}{2} \log \frac{\alpha P+N_{1}}{N_{1}}-I\left(\mathbf{S}_{1}^{n}, \mathbf{S}_{2}^{n} ; Y_{1}^{n} \mid \mathbf{V}_{1}^{n}, \mathbf{V}_{2}^{n}\right) \\
& =\frac{n}{2} \log \frac{\alpha P+N_{1}}{N_{1}}-\frac{n}{2} \log \frac{\left|2 \pi e \Sigma_{\mathbf{S}_{1}, \mathbf{S}_{2}}\right|\left|2 \pi e \Sigma_{\mathbf{U}_{1}, \mathbf{U}_{2}}\right|}{\left|2 \pi e\left(\Sigma_{\mathbf{S}_{1}, \mathbf{S}_{2}}+\Sigma_{\mathbf{U}_{1}, \mathbf{U}_{2}}\right)\right|} \\
& +h\left(\mathbf{S}_{1}^{n}, \mathbf{S}_{2}^{n} \mid \mathbf{V}_{1}^{n}, \mathbf{V}_{2}^{n}, Y_{1}^{n}\right) \\
& =\frac{n}{2} \log \frac{\alpha P+N_{1}}{N_{1}}-\frac{n}{2} \log \frac{\left|2 \pi e \Sigma_{\mathbf{S}_{1}, \mathbf{S}_{2}}\right|\left|2 \pi e \Sigma_{\mathbf{U}_{1}, \mathbf{U}_{2}}\right|}{\left|2 \pi e\left(\Sigma_{\mathbf{S}_{1}, \mathbf{S}_{2}}+\Sigma_{\mathbf{U}_{1}, \mathbf{U}_{2}}\right)\right|} \\
& +h\left(\mathbf{S}_{1}^{n}-\hat{\mathbf{S}}_{1}^{n}, \mathbf{S}_{2}^{n}-\tilde{\mathbf{S}}_{2}^{n} \mid \mathbf{V}_{1}^{n}-\hat{\mathbf{S}}_{1}^{n}, \mathbf{V}_{2}^{n}-\tilde{\mathbf{S}}_{2}^{n}, Y_{1}^{n}\right) \\
& \leq \frac{n}{2} \log \frac{\alpha P+N_{1}}{N_{1}}-\frac{n}{2} \log \frac{\left|2 \pi e \Sigma_{\mathbf{S}_{1}, \mathbf{S}_{2}}\right|\left|2 \pi e \Sigma_{\mathbf{U}_{1}, \mathbf{U}_{2}}\right|}{\left|2 \pi e\left(\Sigma_{\mathbf{S}_{1}, \mathbf{S}_{2}}+\Sigma_{\mathbf{U}_{1}, \mathbf{U}_{2}}\right)\right|} \\
& +h\left(\mathbf{S}_{1}^{n}-\hat{\mathbf{S}}_{1}^{n}, \mathbf{S}_{2}^{n}-\tilde{\mathbf{S}}_{2}^{n} \mid \mathbf{V}_{1}^{n}-\hat{\mathbf{S}}_{1}^{n}, \mathbf{V}_{2}^{n}-\tilde{\mathbf{S}}_{2}^{n}\right) \\
& \leq \frac{n}{2} \log \frac{\alpha P+N_{1}}{N_{1}}-\frac{n}{2} \log \frac{\left|\Sigma_{\mathbf{S}_{1}, \mathbf{S}_{2}}\right|\left|\Sigma_{\mathbf{U}_{1}, \mathbf{U}_{2}}\right|}{\left|\Sigma_{\mathbf{S}_{1}, \mathbf{S}_{2}}+\Sigma_{\mathbf{U}_{1}, \mathbf{U}_{2}}\right|} \\
& +\frac{n}{2} \log \frac{|\boldsymbol{\Theta}|\left|\Sigma_{\mathbf{U}_{1}, \mathbf{U}_{2}}\right|}{\left|\boldsymbol{\Theta}+\Sigma_{\mathbf{U}_{1}, \mathbf{U}_{2}}\right|} \\
& =\frac{n}{2} \log \frac{\left(\alpha P+N_{1}\right)\left|\Sigma_{\mathbf{S}_{1}, \mathbf{S}_{2}}+\Sigma_{\mathbf{U}_{1}, \mathbf{U}_{2}}\right||\boldsymbol{\Theta}|}{N_{1}\left|\Sigma_{\mathbf{S}_{1}, \mathbf{S}_{2}}\right|\left|\boldsymbol{\Theta}+\Sigma_{\mathbf{U}_{1}, \mathbf{U}_{2}}\right|},
\end{aligned}
$$

where (10) is due to Lemma 2. On the other hand,

$$
\begin{aligned}
& I\left(\mathbf{V}_{1}^{n} ; Y_{1}^{n} \mid \mathbf{V}_{2}^{n}\right) \\
& =h\left(\mathbf{V}_{1}^{n} \mid \mathbf{V}_{2}^{n}\right)-h\left(\mathbf{V}_{1}^{n} \mid \mathbf{V}_{2}^{n}, Y_{1}^{n}\right) \\
& =\frac{n}{2} \log \frac{\left|2 \pi e\left(\Sigma_{\mathbf{S}_{1}, \mathbf{S}_{2}}+\Sigma_{\mathbf{U}_{1}, \mathbf{U}_{2}}\right)\right|}{\left|2 \pi e\left(\Sigma_{\mathbf{S}_{2}}+\Sigma_{\mathbf{U}_{2}}\right)\right|}-h\left(\mathbf{V}_{1}^{n} \mid \mathbf{V}_{2}^{n}, Y_{1}^{n}\right) \\
& =\frac{n}{2} \log \frac{\left|2 \pi e\left(\Sigma_{\mathbf{S}_{1}, \mathbf{S}_{2}}+\Sigma_{\mathbf{U}_{1}, \mathbf{U}_{2}}\right)\right|}{\left|2 \pi e\left(\Sigma_{\mathbf{S}_{2}}+\Sigma_{\mathbf{U}_{2}}\right)\right|}-h\left(\mathbf{V}_{1}^{n}-\hat{\mathbf{S}}_{1}^{n} \mid \mathbf{V}_{2}^{n}-\tilde{\mathbf{S}}_{2}^{n}, Y_{1}^{n}\right) \\
& \geq \frac{n}{2} \log \frac{\left|2 \pi e\left(\Sigma_{\mathbf{S}_{1}, \mathbf{S}_{2}}+\Sigma_{\mathbf{U}_{1}, \mathbf{U}_{2}}\right)\right|}{\left|2 \pi e\left(\Sigma_{\mathbf{S}_{2}}+\Sigma_{\mathbf{U}_{2}}\right)\right|}-h\left(\mathbf{V}_{1}^{n}-\hat{\mathbf{S}}_{1}^{n} \mid \mathbf{V}_{2}^{n}-\tilde{\mathbf{S}}_{2}^{n}\right) \\
& \geq \frac{n}{2} \log \frac{\left|2 \pi e\left(\Sigma_{\mathbf{S}_{1}, \mathbf{S}_{2}}+\Sigma_{\mathbf{U}_{1}, \mathbf{U}_{2}}\right)\right|}{\left|2 \pi e\left(\Sigma_{\mathbf{S}_{2}}+\Sigma_{\mathbf{U}_{2}}\right)\right|}-\frac{n}{2} \log \frac{\left|2 \pi e\left(\boldsymbol{\Theta}+\Sigma_{\mathbf{U}_{1}, \mathbf{U}_{2}}\right)\right|}{\left|2 \pi e\left(\boldsymbol{\Theta}_{2}+\Sigma_{\mathbf{U}_{2}}\right)\right|} \\
& =\frac{n}{2} \log \frac{\left|\Sigma_{\mathbf{S}_{1}, \mathbf{S}_{2}}+\Sigma_{\mathbf{U}_{1}, \mathbf{U}_{2}}\right|\left|\mathbf{\Theta}_{2}+\Sigma_{\mathbf{U}_{2}}\right|}{\left|\Sigma_{\mathbf{S}_{2}}+\Sigma_{\mathbf{U}_{2}}\right|\left|\mathbf{\Theta}_{\mathbf{S}_{1}, \mathbf{U}_{2}}\right|},
\end{aligned}
$$

where (12) follows from Lemma 2. Combining (11) and (13) yields

$$
\frac{\left|\boldsymbol{\Theta}_{2}+\Sigma_{\mathbf{U}_{2}}\right|}{\left|\Sigma_{\mathbf{S}_{2}}+\Sigma_{\mathbf{U}_{2}}\right|} \leq \frac{\left(\alpha P+N_{1}\right)|\boldsymbol{\Theta}|}{N_{1}\left|\Sigma_{\mathbf{S}_{1}, \mathbf{S}_{2}}\right|} .
$$

One can readily obtain (4) from (8) and (14) by eliminating $\alpha$. This completes the proof of Theorem 1.

This theorem leads us to the following (potentially weakened) lower bound on $P\left(\mathbf{D}_{1}, \mathbf{D}_{2}\right)$. Somewhat surprisingly, this lower bound turns out to be tight in the vector-scalar case.

Corollary 1:

$$
\begin{aligned}
P\left(\mathbf{D}_{1}, \mathbf{D}_{2}\right) \geq & \sup _{\Sigma_{\mathbf{U}_{1}, \mathbf{U}_{2} \succ \mathbf{0}}} N_{1} \frac{\left|\Sigma_{\mathbf{S}_{1}, \mathbf{S}_{2}}+\Sigma_{\mathbf{U}_{1}, \mathbf{U}_{2}}\right|}{\left|\mathbf{D}_{1}+\Sigma_{\mathbf{U}_{1}}\right|\left|\mathbf{D}_{2}+\Sigma_{\mathbf{U}_{2}}\right|} \\
& +\left(N_{2}-N_{1}\right) \frac{\left|\Sigma_{\mathbf{S}_{2}}+\Sigma_{\mathbf{U}_{2}}\right|}{\left|\mathbf{D}_{2}+\Sigma_{\mathbf{U}_{2}}\right|}-N_{2} .
\end{aligned}
$$

Proof of Corollary 1: Note that

$$
\frac{\left|\Sigma_{\mathbf{S}_{1}, \mathbf{S}_{2}}\right|\left|\boldsymbol{\Theta}_{2}+\Sigma_{\mathbf{U}_{2}}\right|}{|\boldsymbol{\Theta}|\left|\mathbf{D}_{2}+\Sigma_{\mathbf{U}_{2}}\right|}=\frac{\left|\Sigma_{\mathbf{S}_{1}, \mathbf{S}_{2}}\right|\left|\boldsymbol{\Theta}_{2}+\Sigma_{\mathbf{U}_{2}}\right|\left|\boldsymbol{\Theta}+\Sigma_{\mathbf{U}_{1}, \mathbf{U}_{2}}\right|}{|\boldsymbol{\Theta}|\left|\mathbf{D}_{2}+\Sigma_{\mathbf{U}_{2}}\right|\left|\boldsymbol{\Theta}+\Sigma_{\mathbf{U}_{1}, \mathbf{U}_{2}}\right|}
$$

For any $\boldsymbol{\Theta}$ satisfying (2) and (3), we have

$$
\begin{aligned}
& \frac{\left|\boldsymbol{\Theta}+\Sigma_{\mathbf{U}_{1}, \mathbf{U}_{2}}\right|}{|\boldsymbol{\Theta}|} \geq \frac{\left|\Sigma_{\mathbf{S}_{1}, \mathbf{S}_{2}}+\Sigma_{\mathbf{U}_{1}, \mathbf{U}_{2}}\right|}{\left|\Sigma_{\mathbf{S}_{1}, \mathbf{S}_{2}}\right|}, \\
& \frac{\left|\boldsymbol{\Theta}_{2}+\Sigma_{\mathbf{U}_{2}}\right|}{\left|\boldsymbol{\Theta}+\Sigma_{\mathbf{U}_{1}, \mathbf{U}_{2}}\right|} \geq \frac{1}{\left|\boldsymbol{\Theta}_{1}+\Sigma_{\mathbf{U}_{1}}\right|} \geq \frac{1}{\left|\mathbf{D}_{1}+\Sigma_{\mathbf{U}_{1}}\right|},
\end{aligned}
$$

where (16) is due to the fact that $\frac{\left|\mathbf{A}_{1}+\mathbf{B}\right|}{\left|\mathbf{A}_{1}\right|} \geq \frac{\left|\mathbf{A}_{2}+\mathbf{B}\right|}{\left|\mathbf{A}_{2}\right|}$ for $\mathbf{A}_{2} \succeq \mathbf{A}_{1} \succ \mathbf{0}$ and $\mathbf{B} \succeq \mathbf{0}$, and the first inequality in (17) is a consequence of Fischer's inequality. Substituting (16) and (17) into (15) yields

$$
\frac{\left|\Sigma_{\mathbf{S}_{1}, \mathbf{S}_{2}}\right|\left|\boldsymbol{\Theta}_{2}+\Sigma_{\mathbf{U}_{2}}\right|}{|\Theta|\left|\mathbf{D}_{2}+\Sigma_{\mathbf{U}_{2}}\right|} \geq \frac{\left|\Sigma_{\mathbf{S}_{1}, \mathbf{S}_{2}}+\Sigma_{\mathbf{U}_{1}, \mathbf{U}_{2}}\right|}{\left|\mathbf{D}_{1}+\Sigma_{\mathbf{U}_{1}}\right|\left|\mathbf{D}_{2}+\Sigma_{\mathbf{U}_{2}}\right|},
$$

from which Corollary 1 follows immediately.

It is also possible to derive this lower bound by taking a shortcut in the proof of Theorem 1. 
Alternative Proof of Corollary 1: Note that

$$
\begin{aligned}
I\left(\mathbf{V}_{1}^{n} ; Y_{1}^{n} \mid\right. & \left.\mathbf{V}_{2}^{n}\right) \\
& =h\left(Y_{1}^{n} \mid \mathbf{V}_{2}^{n}\right)-h\left(Y_{1}^{n} \mid \mathbf{V}_{1}^{n}, \mathbf{V}_{2}^{n}\right) \\
& \leq \frac{n}{2} \log \left(2 \pi e\left(\alpha P+N_{1}\right)\right)-h\left(Y_{1}^{n} \mid \mathbf{S}_{1}^{n}, \mathbf{S}_{2}^{n}\right) \\
& =\frac{n}{2} \log \left(2 \pi e\left(\alpha P+N_{1}\right)\right)-h\left(Z_{1}^{n}\right) \\
& =\frac{n}{2} \log \frac{\alpha P+N_{1}}{N_{1}} .
\end{aligned}
$$

On the other hand,

$$
\begin{aligned}
& I\left(\mathbf{V}_{1}^{n} ; Y_{1}^{n} \mid \mathbf{V}_{2}^{n}\right) \\
& \quad=h\left(\mathbf{V}_{1}^{n} \mid \mathbf{V}_{2}^{n}\right)-h\left(\mathbf{V}_{1}^{n} \mid \mathbf{V}_{2}^{n}, Y_{1}^{n}\right) \\
& \quad=\frac{n}{2} \log \frac{\left|2 \pi e\left(\Sigma_{\mathbf{S}_{1}, \mathbf{S}_{2}}+\Sigma_{\mathbf{U}_{1}, \mathbf{U}_{2}}\right)\right|}{\left|2 \pi e\left(\Sigma_{\mathbf{S}_{2}}+\Sigma_{\mathbf{U}_{2}}\right)\right|}-h\left(\mathbf{V}_{1}^{n} \mid \mathbf{V}_{2}^{n}, Y_{1}^{n}\right) \\
& \quad \geq \frac{n}{2} \log \frac{\left|2 \pi e\left(\Sigma_{\mathbf{S}_{1}, \mathbf{S}_{2}}+\Sigma_{\mathbf{U}_{1}, \mathbf{U}_{2}}\right)\right|}{\left|2 \pi e\left(\Sigma_{\mathbf{S}_{2}}+\Sigma_{\mathbf{U}_{2}}\right)\right|}-h\left(\mathbf{V}_{1}^{n} \mid Y_{1}^{n}\right) \\
& \quad \geq \frac{n}{2} \log \frac{\left|2 \pi e\left(\Sigma_{\mathbf{S}_{1}, \mathbf{S}_{2}}+\Sigma_{\mathbf{U}_{1}, \mathbf{U}_{2}}\right)\right|}{\left|2 \pi e\left(\Sigma_{\mathbf{S}_{2}}+\Sigma_{\mathbf{U}_{2}}\right)\right|}-h\left(\mathbf{V}_{1}^{n} \mid \hat{\mathbf{S}}_{1}^{n}\right) \\
& \quad \geq \frac{n}{2} \log \frac{\left|2 \pi e\left(\Sigma_{\mathbf{S}_{1}, \mathbf{S}_{2}}+\Sigma_{\mathbf{U}_{1}, \mathbf{U}_{2}}\right)\right|}{\left|2 \pi e\left(\Sigma_{\mathbf{S}_{2}}+\Sigma_{\mathbf{U}_{2}}\right)\right|}-\frac{n}{2} \log \left|2 \pi e\left(\mathbf{\Theta}_{1}+\Sigma_{\mathbf{U}_{1}}\right)\right| \\
& \geq \frac{n}{2} \log \frac{\left|\Sigma_{\mathbf{S}_{1}, \mathbf{S}_{2}}+\Sigma_{\mathbf{U}_{1}, \mathbf{U}_{2}}\right|}{\left|\Sigma_{\mathbf{S}_{2}}+\Sigma_{\mathbf{U}_{2}}\right|\left|\mathbf{D}_{1}+\Sigma_{\mathbf{U}_{1}}\right|},
\end{aligned}
$$

where (21) follows from Lemma 1. Combining (19) and (22) yields

$$
\frac{\left|\Sigma_{\mathbf{S}_{1}, \mathbf{S}_{2}}+\Sigma_{\mathbf{U}_{1}, \mathbf{U}_{2}}\right|}{\left|\Sigma_{\mathbf{S}_{2}}+\Sigma_{\mathbf{U}_{2}}\right|\left|\mathbf{D}_{1}+\Sigma_{\mathbf{U}_{1}}\right|} \leq \frac{\alpha P+N_{1}}{N_{1}}
$$

which, together with (8), proves Corollary 1 .

In order for the inequalities in (18) and (20) to become equalities, we need to have

$$
\begin{aligned}
I\left(\mathbf{V}_{1}^{n}, \mathbf{V}_{2}^{n} ; Y_{1}^{n}\right) & =I\left(\mathbf{S}_{1}^{n}, \mathbf{S}_{2}^{n} ; Y_{1}^{n}\right), \\
I\left(\mathbf{V}_{1}^{n} ; \mathbf{V}_{2}^{n} \mid Y_{1}^{n}\right) & =0 .
\end{aligned}
$$

It will be seen that these two conditions provide important guidelines for constructing hybrid schemes that achieve the lower bound in Corollary 1 . Note that the derivation of this lower bound is based on a consideration of the scenario where $\mathbf{V}_{2}$ is provided to the strong receiver by a genie. Intuitively, a necessary condition for this lower bound to be tight is that the side information provided by the genie is superfluous, which is exactly the implication of (24).

\section{The Vector-Scalar CASE}

We shall show in this section that the lower bound in Corollary 1 is tight for the vector-scalar case, i.e., the scenario where the weak receiver wishes to reconstruct a scalar source (i.e., $m_{2}=1$ ) under the mean squared error distortion constraint. In this special setup, we denote $\mathbf{S}_{2}, \Sigma_{\mathbf{S}_{2}}, \mathbf{D}_{2}, \mathbf{U}_{2}, \Sigma_{\mathbf{U}_{2}}$ by $S_{2}, \sigma_{S_{2}}^{2}, d_{2}, U_{2}, \sigma_{U_{2}}^{2}$, respectively.
Theorem 2:

$$
\begin{aligned}
P\left(\mathbf{D}_{1}, d_{2}\right)= & \sup _{\Sigma_{\mathbf{U}_{1}, U_{2}} \succ \mathbf{0}} N_{1} \frac{\left|\Sigma_{\mathbf{S}_{1}, S_{2}}+\Sigma_{\mathbf{U}_{1}, U_{2}}\right|}{\left|\mathbf{D}_{1}+\Sigma_{\mathbf{U}_{1}}\right|\left(d_{2}+\sigma_{U_{2}}^{2}\right)} \\
& +\left(N_{2}-N_{1}\right) \frac{\sigma_{S_{2}}^{2}+\sigma_{U_{2}}^{2}}{d_{2}+\sigma_{U_{2}}^{2}}-N_{2} .
\end{aligned}
$$

\section{A. Upper Bound}

Proof of Theorem 2: To the end of proving Theorem 2, it suffices to show that the right-hand side of (25) is (asymptotically) achievable and consequently is an upper bound on $P\left(\mathbf{D}_{1}, d_{2}\right)$. Our achievability argument is based on a hybrid scheme, which bears some resemblance to the one proposed by Puri et al. in a different setting [18] (see also [19]). It will be seen that this hybrid scheme is semi-universal in the sense that the encoder only needs to know $N_{1}$ but not $N_{2}$. Let us first introduce a zero-mean random vector $\mathbf{S}_{1}(\gamma)$ and a zero-mean random variable $S_{2}(\gamma)$ that are jointly Gaussian. They are related with $\left(\mathbf{S}_{1}, S_{2}\right)$ via a backward Gaussian test channel $\left(\mathbf{S}_{1}, S_{2}\right)=\left(\mathbf{S}_{1}(\gamma)+\mathbf{Q}_{1}, S_{2}(\gamma)+Q_{2}\right)$, where $\left(\mathbf{Q}_{1}, Q_{2}\right)$ is independent of $\left(\mathbf{S}_{1}(\gamma), S_{2}(\gamma)\right)$. The covariance matrix of $\left(\mathbf{S}_{1}(\gamma), S_{2}(\gamma)\right)$, parametrized by a scalar variable $\gamma$, is to be specified later. We assume that $\left(\mathbf{S}_{1}, S_{2}, \mathbf{S}_{1}(\gamma), S_{2}(\gamma)\right)$ is independent of $\left(Z_{1}, Z_{2}\right)$. Note that we can write

$$
\begin{aligned}
\mathbf{S}_{1}(\gamma) & =\mathbb{E}\left[\mathbf{S}_{1}(\gamma) \mid \mathbf{S}_{1}, S_{2}, S_{2}(\gamma)\right]+\mathbf{W}_{1} \\
& =\mathbf{A}_{1} \mathbf{S}_{1}+\mathbf{a}_{2} S_{2}+\mathbf{a}_{3} S_{2}(\gamma)+\mathbf{W}_{1}, \\
S_{2}(\gamma) & =\mathbb{E}\left[S_{2}(\gamma) \mid \mathbf{S}_{1}, S_{2}\right]+W_{2} \\
& =\mathbf{b}_{1}^{T} \mathbf{S}_{1}+b_{2} S_{2}+W_{2},
\end{aligned}
$$

where $\mathbf{W}_{1}$ is independent of $\left(\mathbf{S}_{1}, S_{2}, S_{2}(\gamma)\right)$, and $W_{2}$ is independent of $\left(\mathbf{S}_{1}, S_{2}\right)$. Next define

$$
\tilde{\mathbf{S}}_{1}(\gamma)=\mathbf{A}_{1} \mathbf{S}_{1}+\mathbf{a}_{2} S_{2}+\mathbf{W}_{1} .
$$

We are now in a position to describe the scheme (see Fig. 2). Since the scheme is a combination of some well-known coding techniques, e.g., Wyner-Ziv codes [20] and dirty paper codes [21], we only provide an outline of the encoding and decoding steps, and then focus on the condition that guarantees correct decoding.

1) Encoding: Let the channel input $X^{n}$, with average power $P(\gamma)$, be a superposition of an analog signal $X_{a}^{n}$ and a digital signal $X_{d}^{n}$ (i.e., $X^{n}=X_{a}^{n}+X_{d}^{n}$ ). The analog portion is given by $X_{a}^{n}=\beta\left(\mathbf{b}_{1}^{T} \mathbf{S}_{1}^{n}+b_{2} S_{2}^{n}\right)$ for some non-negative number $\beta$ to be specified later. For the digital portion $X_{d}^{n}$, the encoder first uses a Wyner-Ziv code of rate $R$ with codewords generated according to $\tilde{\mathbf{S}}_{1}(\gamma)$, with $\left(\mathbf{S}_{1}^{n}, S_{2}^{n}\right)$ as the input, and with $Y_{1}^{n} \triangleq X_{a}^{n}+X_{d}^{n}+Z_{1}^{n}$ as the decoder side information; the encoder then determines the digital portion of the channel input $X_{d}^{n}$ to send the bin index of the chosen Wyner-Ziv codeword $\tilde{\mathbf{S}}_{1}^{n}(\gamma)$ by using a dirty paper code of rate $R$ with $X_{a}^{n}$ treated as the channel state information known at the encoder. We define $P_{a}=\mathbb{E}\left[\left(X_{a}\right)^{2}\right]$ and $P_{d}=\mathbb{E}\left[\left(X_{d}\right)^{2}\right]$, where $X_{a} \triangleq \beta\left(\mathbf{b}_{1}^{T} \mathbf{S}_{1}+b_{2} S_{2}\right)$ and $X_{d}$ are mutually independently zero-mean Gaussian random variables, and $P_{a}+P_{d}=P(\gamma)$. 

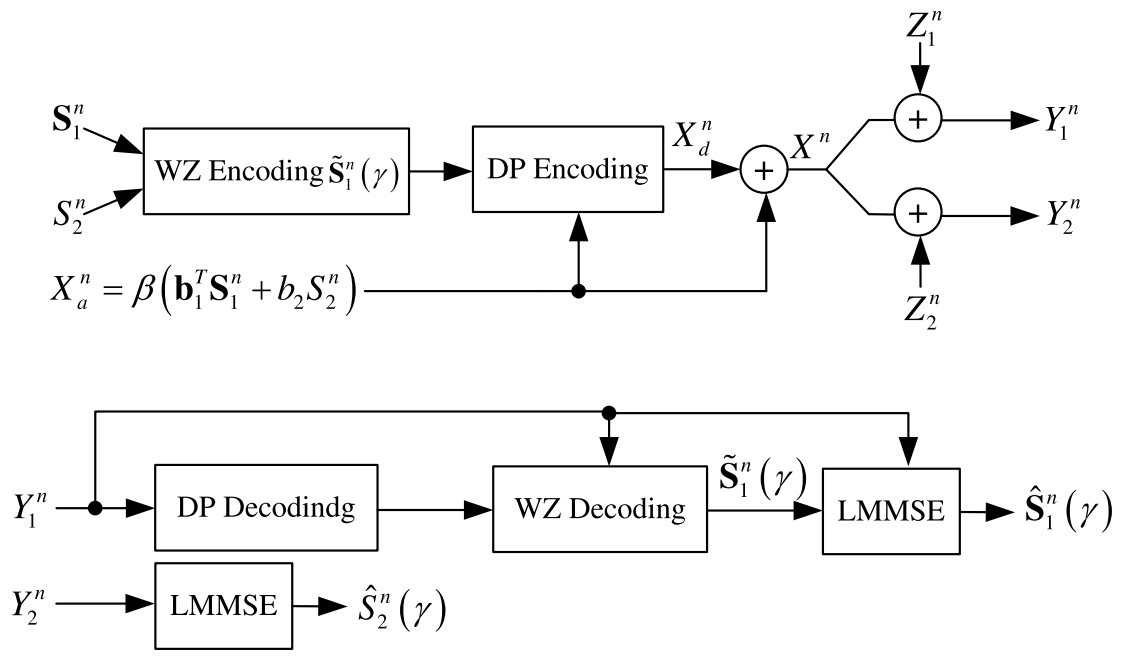

Fig. 2. An illustration of the hybrid scheme in Section IV-A.

2) Decoding: Receiver 1 first decodes the dirty paper code; it then further recovers $\tilde{\mathbf{S}}_{1}^{n}(\gamma)$ by decoding the Wyner-Ziv code with $Y_{1}^{n}$ as the side information. In view of the fact that the linear MMSE estimate of $\mathbf{S}_{1}$ based on $\tilde{\mathbf{S}}_{1}(\gamma)$ and $Y_{1} \triangleq X_{a}+$ $X_{d}+Z_{1}$ is $\hat{\mathbf{S}}_{1}(\gamma) \triangleq \tilde{\mathbf{S}}_{1}(\gamma)+\beta^{-1} \mathbf{a}_{3} Y_{1}$, Receiver 1 can use $\hat{\mathbf{S}}_{1}^{n}(\gamma) \triangleq \tilde{\mathbf{S}}_{1}^{n}(\gamma)+\beta^{-1} \mathbf{a}_{3} Y_{1}^{n}$ as the reconstruction of $\mathbf{S}_{1}^{n}$. Since the linear MMSE estimate of $S_{2}$ based on $Y_{2} \triangleq X_{a}+X_{d}+Z_{2}$ is $\hat{S}_{2}(\gamma) \triangleq \rho Y_{2}$ with $\rho=\mathbb{E}\left[S_{2} X_{a}\right]\left(P(\gamma)+N_{2}\right)^{-1}$, Receiver 2 can simply use $\hat{S}_{2}^{n}(\gamma) \triangleq \rho Y_{2}^{n}$ as the reconstruction of $S_{2}^{n}$, where $Y_{2}^{n}=X_{a}^{n}+X_{d}^{n}+Z_{2}^{n}$; the resulting distortion is denoted by $d_{2}(\gamma)$.

3) Coding Parameters: For a given covariance matrix of $\left(\mathbf{S}_{1}(\gamma), S_{2}(\gamma)\right)$, three parameters $\beta, P_{d}$, and $R$ still need to be specified for the aforedescribed scheme. Equivalently, we shall specify $\beta, P(\gamma)$, and $R$, since $\beta$ determines $P_{a}$ and $P_{d}=P(\gamma)-P_{a}$. Let us first choose $P(\gamma)$ such that

$$
I\left(\mathbf{S}_{1}, S_{2} ; \mathbf{S}_{1}(\gamma), S_{2}(\gamma)\right)=\frac{1}{2} \log \frac{P(\gamma)+N_{1}}{N_{1}} .
$$

The parameter $\beta$ is then chosen such that

$$
I\left(X_{a} ; Y_{1}\right)=I\left(\mathbf{S}_{1}, S_{2} ; S_{2}(\gamma)\right),
$$

which is always possible because

$$
\begin{aligned}
I\left(\mathbf{S}_{1}, S_{2} ; S_{2}(\gamma)\right) & \leq I\left(\mathbf{S}_{1}, S_{2} ; \mathbf{S}_{1}(\gamma), S_{2}(\gamma)\right) \\
& =\frac{1}{2} \log \frac{P(\gamma)+N_{1}}{N_{1}},
\end{aligned}
$$

and one can let $I\left(X_{a} ; Y_{1}\right)$ take any value in $\left[0, \frac{1}{2} \log \frac{P(\gamma)+N_{1}}{N_{1}}\right]$ by varying $\beta$. Finally set

$$
R=I\left(\mathbf{S}_{1}, S_{2} ; \tilde{\mathbf{S}}_{1}(\gamma) \mid Y_{1}\right)
$$

Now the scheme is fully specified for any given covariance matrix of $\left(\mathbf{S}_{1}(\gamma), S_{2}(\gamma)\right)$.

4) Conditions for Correct Decoding: The Wyner-Ziv code and the dirty paper code need to be decoded correctly at Receiver 1. It is easily seen that the Wyner-Ziv code is ensured to be decoded correctly by (28), and thus we focus on the decodability of dirty paper code. First note that (27), together with the fact that $I\left(X_{a} ; Y_{1}\right)=I\left(\mathbf{S}_{1}, S_{2} ; Y_{1}\right)$, implies that $I\left(\mathbf{S}_{1}, S_{2} ; Y_{1}\right)=I\left(\mathbf{S}_{1}, S_{2} ; S_{2}(\gamma)\right)$; moreover, since both $X_{d}+Z_{1}$ and $W_{2}$, which are Gaussian random variables, are independent of $\left(\mathbf{S}_{1}, S_{2}\right)$, it follows that the joint distributions of $\left(\mathbf{S}_{1}, S_{2}, \beta^{-1} Y_{1}\right)$ and $\left(\mathbf{S}_{1}, S_{2}, S_{2}(\gamma)\right)$ are identical, which, in view of the fact that $\mathbf{W}_{1}$ is independent of $\left(\mathbf{S}_{1}, S_{2}, S_{2}(\gamma), Y_{1}\right)$, further implies that the joint distributions of $\left(\mathbf{S}_{1}, S_{2}, \tilde{\mathbf{S}}_{1}(\gamma), \beta^{-1} Y_{1}\right)$ and $\left(\mathbf{S}_{1}, S_{2}, \tilde{\mathbf{S}}_{1}(\gamma), S_{2}(\gamma)\right)$ are identical. $^{2}$ Therefore, we have

$$
R=I\left(\mathbf{S}_{1}, S_{2} ; \tilde{\mathbf{S}}_{1}(\gamma) \mid S_{2}(\gamma)\right)
$$

Furthermore, note that

$$
\begin{aligned}
I\left(\mathbf{S}_{1}, S_{2} ;\right. & \left.\tilde{\mathbf{S}}_{1}(\gamma) \mid S_{2}(\gamma)\right) \\
& =I\left(\mathbf{S}_{1}, S_{2} ; \tilde{\mathbf{S}}_{1}(\gamma), S_{2}(\gamma)\right)-I\left(\mathbf{S}_{1}, S_{2} ; S_{2}(\gamma)\right) \\
& =I\left(\mathbf{S}_{1}, S_{2} ; \mathbf{S}_{1}(\gamma), S_{2}(\gamma)\right)-I\left(X_{a} ; Y_{1}\right) \\
& =\frac{1}{2} \log \frac{P(\gamma)+N_{1}}{N_{1}}-\frac{1}{2} \log \frac{P(\gamma)+N_{1}}{P_{d}+N_{1}} \\
& =\frac{1}{2} \log \frac{P_{d}+N_{1}}{N_{1}},
\end{aligned}
$$

which, together with (29), ensures that Receiver 1 can correctly decode the dirty paper code.

5) Optimizing the Covariance Matrix of $\left(\boldsymbol{S}_{1}(\gamma), S_{2}(\gamma)\right)$ : Now only the covariance matrix of $\left(\mathbf{S}_{1}(\gamma), S_{2}(\gamma)\right)$ remains to be specified. To this end we formulate the following maximization problem. It will become clear that this maximization problem is motivated by the lower bound in Corollary 1. In particular, it will be seen that the hybrid scheme and the remote sources induced by the optimal solution (and the associated Lagrangian multipliers) of this maximization problem possess the desired properties (see (23) and (24)).

\footnotetext{
${ }^{2}$ We have implicitly assumed that $\mathbb{E}\left[\left(\mathbf{b}_{1}^{T} \mathbf{S}_{1}+b_{2} S_{2}\right)^{2}\right]>0$ (which implies that the $P_{a}$ and the $\beta$ determined by (27) are positive). For the degenerate case $\mathbf{b}_{1}^{T} \mathbf{S}_{1}+b_{2} S_{2}=0$ (which is possible if and only if $S_{2}(\gamma)=0$ ), one can simply set $X_{a}=0$ and $\beta^{-1} Y_{1}=0$.
} 
Given $\gamma \in(0, \infty)$, let $\boldsymbol{\Theta}(\gamma)$ denote the solution ${ }^{3}$ to

$$
\begin{aligned}
& \max _{\boldsymbol{\Theta}} \log |\boldsymbol{\Theta}| \\
& \text { subject to } \boldsymbol{\Theta}_{1} \preceq \mathbf{D}_{1}, \\
& \\
& \theta_{2} \leq \gamma, \\
& \mathbf{0} \preceq \mathbf{\Theta} \preceq \Sigma_{\mathbf{S}_{1}, S_{2}},
\end{aligned}
$$

where $\boldsymbol{\Theta}_{1}$ is the first $m_{1} \times m_{1}$ diagonal submatrix of $\boldsymbol{\Theta}$, and $\theta_{2}$ is the $\left(m_{1}+1, m_{1}+1\right)$ entry of $\boldsymbol{\Theta}$. It can be shown (see Appendix C) that $\boldsymbol{\Theta}(\gamma)$ is a continuous function of $\gamma$. We denote the first $m_{1} \times m_{1}$ diagonal submatrix of $\boldsymbol{\Theta}(\gamma)$ by $\boldsymbol{\Theta}_{1}(\gamma)$, and the $\left(m_{1}+1, m_{1}+1\right)$ entry of $\boldsymbol{\Theta}(\gamma)$ by $\theta_{2}(\gamma)$. Now choose the covariance matrix of $\left(\mathbf{S}_{1}(\gamma), S_{2}(\gamma)\right)$ to be $\Sigma_{\mathbf{S}_{1}, S_{2}}-\boldsymbol{\Theta}(\gamma)$; as a consequence, the covariance matrix of $\mathbf{S}_{1}(\gamma)$ is $\Sigma_{\mathbf{S}_{1}}-\mathbf{\Theta}_{1}(\gamma)$, and the variance of $S_{2}(\gamma)$ is $\sigma_{S_{2}}^{2}-\theta_{2}(\gamma)$. Accordingly, (26) reduces to

$$
\frac{1}{2} \log \frac{\left|\Sigma_{\mathbf{S}_{1}, S_{2}}\right|}{|\boldsymbol{\Theta}(\gamma)|}=\frac{1}{2} \log \frac{P(\gamma)+N_{1}}{N_{1}} .
$$

6) Evaluating the Distortions and the Transmit Power: For the distortion at Receiver 1, it is readily seen that

$$
\begin{aligned}
\mathbb{E}\left[\left(\mathbf{S}_{1}\right.\right. & \left.\left.-\hat{\mathbf{S}}_{1}(\gamma)\right)\left(\mathbf{S}_{1}-\hat{\mathbf{S}}_{1}(\gamma)\right)^{T}\right] \\
& =\mathbb{E}\left[\left(\mathbf{S}_{1}-\mathbf{S}_{1}(\gamma)\right)\left(\mathbf{S}_{1}-\mathbf{S}_{1}(\gamma)\right)^{T}\right] \\
& =\boldsymbol{\Theta}_{1}(\gamma) \\
& \preceq \mathbf{D}_{1},
\end{aligned}
$$

where (32) is true because the joint distributions of $\left(\mathbf{S}_{1}, \hat{\mathbf{S}}_{1}(\gamma)\right)$ and $\left(\mathbf{S}_{1}, \mathbf{S}_{1}(\gamma)\right)$ are identical (which is further due to the fact that the joint distributions of $\left(\mathbf{S}_{1}, \tilde{\mathbf{S}}_{1}(\gamma), \beta^{-1} Y_{1}\right)$ and $\left(\mathbf{S}_{1}, \tilde{\mathbf{S}}_{1}(\gamma), S_{2}(\gamma)\right)$ are identical). It is worth noting that the linear MMSE estimate of $\left(\mathbf{S}_{1}, S_{2}\right)$ based on $\left(\tilde{\mathbf{S}}_{1}(\gamma), Y_{1}\right)$ is $\left(\hat{\mathbf{S}}_{1}(\gamma), \beta^{-1} Y_{1}\right)$. In view of this fact, Receiver 1 can use $\left(\hat{\mathbf{S}}_{1}^{n}(\gamma), \beta^{-1} Y_{1}^{n}\right)$ as the reconstruction of $\left(\mathbf{S}_{1}^{n}, S_{2}^{n}\right)$. Since the joint distributions of $\left(\mathbf{S}_{1}, S_{2}, \hat{\mathbf{S}}_{1}(\gamma), \beta^{-1} Y_{1}\right)$ and $\left(\mathbf{S}_{1}, S_{2}, \mathbf{S}_{1}(\gamma), S_{2}(\gamma)\right)$ are identical, we have

$$
\begin{aligned}
\mathbb{E}\left[\left(\mathbf{S}_{1}^{T}-\hat{\mathbf{S}}_{1}^{T}(\gamma), S_{2}^{T}-\beta^{-1} Y_{1}^{T}\right)^{T}\right. & \left.\left(\mathbf{S}_{1}^{T}-\hat{\mathbf{S}}_{1}^{T}(\gamma), S_{2}^{T}-\beta^{-1} Y_{1}^{T}\right)\right] \\
= & \mathbb{E}\left[\left(\mathbf{S}_{1}^{T}-\hat{\mathbf{S}}_{1}^{T}(\gamma), S_{2}^{T}-S_{2}^{T}(\gamma)\right)^{T}\right. \\
& \left.\quad\left(\mathbf{S}_{1}^{T}-\hat{\mathbf{S}}_{1}^{T}(\gamma), S_{2}^{T}-S_{2}^{T}(\gamma)\right)\right] \\
= & \boldsymbol{\Theta}(\gamma) .
\end{aligned}
$$

Therefore, $\gamma$ can be interpreted as an auxiliary constraint on the reconstruction distortion for $S_{2}^{n}$ at Receiver 1, and $\boldsymbol{\Theta}(\gamma)$ is the actual covariance distortion achieved at Receiver 1 for reconstructing $\left(\mathbf{S}_{1}^{n}, S_{2}^{n}\right)$.

Note that $P(\gamma)$ is a continuous function of $\boldsymbol{\Theta}(\gamma)$ (which is implied by (31)) and consequently is a continuous function of

\footnotetext{
${ }^{3}$ Note that $\boldsymbol{\Theta}(\gamma)$ must be positive definite. Since $\log |\cdot|$ is strictly concave over the domain of positive definite matrices, it follows that $\boldsymbol{\Theta}(\gamma)$ is uniquely defined.
}

$\gamma$ for $\gamma \in(0, \infty)$. Moreover, it can be verified that

$$
\begin{aligned}
& \frac{1}{2} \log \frac{\sigma_{S_{2}}^{2}}{d_{2}(\gamma)} \\
&= \\
&=\frac{1}{2}\left(S_{2} ; Y_{2}\right) \\
&=\frac{P(\gamma)+N_{2}}{\mathbb{E}\left[\left(Y_{2}-\mathbb{E}\left[Y_{2} \mid S_{2}\right]\right)^{2}\right]} \\
&=\frac{P(\gamma)+N_{2}}{2} \log \frac{1}{\mathbb{E}\left[\left(Y_{1}-\mathbb{E}\left[Y_{1} \mid S_{2}\right]\right)^{2}\right]+N_{2}-N_{1}} \\
&=\frac{1}{2} \log \left(P(\gamma)+N_{2}\right)-\frac{1}{2} \log \left(\frac{1}{2 \pi e} e^{2 h\left(Y_{1} \mid S_{2}\right)}+N_{2}-N_{1}\right) \\
&=\frac{1}{2} \log \left(P(\gamma)+N_{2}\right) \\
&-\frac{1}{2} \log \left(\frac{1}{2 \pi e} e^{2\left(h\left(Y_{1}\right)-I\left(S_{2} ; Y_{1}\right)\right)}+N_{2}-N_{1}\right) \\
&= \frac{1}{2} \log \left(P(\gamma)+N_{2}\right) \\
&-\frac{1}{2} \log \left(\frac{P(\gamma)+N_{1}}{2 \pi e \sigma_{S_{2}}^{2}} e^{2 h\left(S_{2} \mid Y_{1}\right)}+N_{2}-N_{1}\right) \\
&= \frac{1}{2} \log \left(P(\gamma)+N_{2}\right) \\
&-\frac{1}{2} \log \left(\frac{\left(P(\gamma)+N_{1}\right) \theta_{2}(\gamma)}{\sigma_{S_{2}}^{2}}+N_{2}-N_{1}\right),
\end{aligned}
$$

where (34) is due to the fact that $h\left(S_{2} \mid Y_{1}\right)=\frac{1}{2} \log \left(2 \pi e \theta_{2}(\gamma)\right)$ (which is implied by (33)). Hence,

$$
d_{2}(\gamma)=\frac{\left(P(\gamma)+N_{1}\right) \theta_{2}(\gamma)+\left(N_{2}-N_{1}\right) \sigma_{S_{2}}^{2}}{P(\gamma)+N_{2}} .
$$

Note that both $P(\gamma)$ and $\theta_{2}(\gamma)$ are continuous in $\gamma$; furthermore, $P(\gamma)$ and $\theta_{2}(\gamma)$ tend to infinity and zero, respectively, as $\gamma \rightarrow 0$. Therefore, $d_{2}(\gamma)$ is a continuous function of $\gamma$ for $\gamma \in(0, \infty)$, and $d_{2}(\gamma)$ tends to zero as $\gamma \rightarrow 0$.

We shall show that

$$
\begin{aligned}
P(\gamma) \leq & \sup _{\Sigma_{\mathbf{U}_{1}, U_{2}} \succ \mathbf{0}} N_{1} \frac{\left|\Sigma_{\mathbf{S}_{1}, S_{2}}+\Sigma_{\mathbf{U}_{1}, U_{2}}\right|}{\left|\mathbf{D}_{1}+\Sigma_{\mathbf{U}_{1}}\right|\left(d_{2}(\gamma)+\sigma_{U_{2}}^{2}\right)} \\
& +\left(N_{2}-N_{1}\right) \frac{\sigma_{S_{2}}^{2}+\sigma_{U_{2}}^{2}}{d_{2}(\gamma)+\sigma_{U_{2}}^{2}}-N_{2}
\end{aligned}
$$

for $\gamma \in(0, \infty)$. To this end we revisit the maximization problem in (30). Note that $\boldsymbol{\Theta}(\gamma)$ must satisfy the following KKT conditions [22]

$$
\begin{aligned}
& \boldsymbol{\Theta}^{-1}(\gamma)-\Lambda-\mathbf{M}=\mathbf{0}, \\
& \Lambda_{1}\left(\mathbf{D}_{1}-\boldsymbol{\Theta}_{1}(\gamma)\right)=\mathbf{0}, \\
& \lambda_{2}\left(\gamma-\theta_{2}(\gamma)\right)=0, \\
& \mathbf{M}\left(\Sigma_{\mathbf{S}_{1}, S_{2}}-\boldsymbol{\Theta}(\gamma)\right)=\mathbf{0},
\end{aligned}
$$

where $\mathbf{M} \succeq \mathbf{0}, \Lambda_{1} \succeq \mathbf{0}, \lambda_{2} \geq 0$, and $\Lambda=\operatorname{diag}\left(\Lambda_{1}, \lambda_{2}\right)$. Let $\Xi_{1} \Pi_{1} \Xi_{1}^{T}$ be the eigenvalue decomposition of $\Lambda_{1}$, where $\Xi_{1}$ is a unitary matrix, and $\Pi_{1}=\operatorname{diag}\left(\pi_{1}, \ldots, \pi_{r}, 0, \ldots, 0\right)$ with $\pi_{i}>0, i=1, \ldots, r$. Define $\Xi=\operatorname{diag}\left(\Xi_{1}, 1\right)$ and $\Pi=\operatorname{diag}\left(\Pi_{1}, \lambda_{2}\right)$. Let $\Pi_{\epsilon}^{\prime}$ be a positive semidefinite diagonal matrix obtained by subtracting $\epsilon$ from each positive diagonal entry of $\Pi$, where $\epsilon$ is an arbitrary positive number smaller than the minimum non-zero diagonal entry of $\Pi$. Since $\boldsymbol{\Theta} \succ \mathbf{0}$, 
it follows that $\Xi^{T} \boldsymbol{\Theta}^{-1}(\gamma) \Xi$ is positive definite. Moreover, in view of (36), we have $\Xi^{T} \boldsymbol{\Theta}^{-1}(\gamma) \Xi-\boldsymbol{\Pi}=\Xi^{T} \mathbf{M} \Xi \succeq \mathbf{0}$. Therefore, $\Xi^{T} \boldsymbol{\Theta}^{-1}(\gamma) \Xi-\Pi_{\epsilon}^{\prime}$ is positive definite when $\epsilon$ is sufficiently small. For any $\epsilon$ with $\Xi^{T} \boldsymbol{\Theta}^{-1}(\gamma) \Xi-\Pi_{\epsilon}^{\prime} \succ \mathbf{0}$, we choose a positive number $\epsilon^{\prime}$, which is a function of $\epsilon$ and tends to zero as $\epsilon \rightarrow 0$, such that

$$
\Xi^{T} \boldsymbol{\Theta}^{-1}(\gamma) \Xi-\Pi_{\epsilon} \succ \mathbf{0},
$$

where $\Pi_{\epsilon}$ is a positive definite diagonal matrix obtained by adding $\epsilon^{\prime}$ to each zero diagonal entry of $\Pi_{\epsilon}^{\prime}$. Now let $\Lambda_{\epsilon}=\Xi \Pi_{\epsilon} \Xi^{T}$ and $\Sigma_{\mathbf{U}_{1, \epsilon}, U_{2, \epsilon}}=\Lambda_{\epsilon}^{-1}-\boldsymbol{\Theta}(\gamma)$. Note that

$$
\begin{gathered}
\Xi^{T} \boldsymbol{\Theta}^{-1}(\gamma) \Xi-\Pi_{\epsilon} \succ \mathbf{0} \\
\Rightarrow \boldsymbol{\Theta}^{-1}(\gamma) \succ \Lambda_{\epsilon} \\
\Rightarrow \Lambda_{\epsilon}^{-1} \succ \boldsymbol{\Theta}(\gamma) .
\end{gathered}
$$

Therefore, $\Sigma_{\mathbf{U}_{1, \epsilon}, U_{2, \epsilon}}$ is positive definite when $\epsilon$ is sufficiently small.

Let $\mathbf{U}_{1, \epsilon}$ and $U_{2, \epsilon}$ be jointly Gaussian with mean zero and covariance matrix $\Sigma_{\mathbf{U}_{1, \epsilon}, U_{2, \epsilon}}$, where $\mathbf{U}_{1, \epsilon}$ is an $m_{1} \times 1$ Gaussian random vector with covariance matrix $\Sigma_{\mathbf{U}_{1, \epsilon}}$ (which is the first $m_{1} \times m_{1}$ diagonal submatrix of $\Sigma_{\mathbf{U}_{1, \epsilon}, U_{2, \epsilon}}$ ) and $U_{2, \epsilon}$ is a Gaussian random variable with variance $\sigma_{U_{2, \epsilon}}^{2}$ (which is the $\left(m_{1}+1, m_{1}+1\right)$ entry of $\left.\Sigma_{\mathbf{U}_{1, \epsilon}, U_{2, \epsilon}}\right)$. We assume that $\left(\mathbf{U}_{1, \epsilon}, U_{2, \epsilon}\right)$ is independent of $\left(\mathbf{S}_{1}, S_{2}, \mathbf{S}_{1}(\gamma), S_{2}(\gamma), Z_{1}, Z_{2}\right)$.

Note that

$$
\begin{aligned}
\lim _{\epsilon \rightarrow 0} \frac{\left|\Sigma_{\mathbf{S}_{1}, S_{2}}+\Sigma_{\mathbf{U}_{1, \epsilon}, U_{2, \epsilon}}\right|}{\mid \boldsymbol{\Theta}(\gamma)}+\Sigma_{\mathbf{U}_{1, \epsilon}, U_{2, \epsilon} \mid} \\
=\lim _{\epsilon \rightarrow 0} \frac{\left|\Sigma_{\mathbf{S}_{1}, S_{2}}+\Lambda_{\epsilon}^{-1}-\boldsymbol{\Theta}(\gamma)\right|}{\left|\Lambda_{\epsilon}^{-1}\right|} \\
=\lim _{\epsilon \rightarrow 0}\left|\Lambda_{\epsilon} \Sigma_{\mathbf{S}_{1}, S_{2}}+\mathbf{I}-\Lambda_{\epsilon} \boldsymbol{\Theta}(\gamma)\right| \\
=\left|\Lambda \Sigma_{\mathbf{S}_{1}, S_{2}}+\mathbf{I}-\Lambda \boldsymbol{\Theta}(\gamma)\right| \\
=\left|\boldsymbol{\Theta}^{-1}(\gamma) \Sigma_{\mathbf{S}_{1}, S_{2}}-\mathbf{M} \Sigma_{\mathbf{S}_{1}, S_{2}}+\mathbf{M} \boldsymbol{\Theta}(\gamma)\right| \\
=\frac{\left|\Sigma_{\mathbf{S}, S_{2}}\right|}{|\boldsymbol{\Theta}(\gamma)|} \\
=\frac{P(\gamma)+N_{1}}{N_{1}},
\end{aligned}
$$

where (38) and (39) are due to (36) and (37), respectively. Moreover, by the definition of $\Sigma_{\mathbf{U}_{1, \epsilon}, U_{2, \epsilon}}$, we have

$$
\boldsymbol{\Theta}(\gamma)+\Sigma_{\mathbf{U}_{1, \epsilon}, U_{2, \epsilon}}=\operatorname{diag}\left(\boldsymbol{\Theta}_{1}(\gamma)+\Sigma_{\mathbf{U}_{1, \epsilon}}, \theta_{2}(\gamma)+\sigma_{U_{2, \epsilon}}^{2}\right) .
$$

It is clear that

$$
\begin{aligned}
I\left(S_{2}+U_{2, \epsilon} ; Y_{2}\right) & =h\left(Y_{2}\right)-h\left(Y_{2} \mid S_{2}+U_{2, \epsilon}\right) \\
& =\frac{1}{2} \log \frac{P(\gamma)+N_{2}}{\alpha_{\epsilon} P(\gamma)+N_{2}},
\end{aligned}
$$

where $\alpha_{\epsilon}=\frac{1}{P(\gamma)} \mathbb{E}\left[\left(X-\mathbb{E}\left[X \mid S_{2}+U_{2, \epsilon}\right]\right)^{2}\right]$. On the other hand,

$$
\begin{aligned}
I\left(S_{2}+U_{2, \epsilon} ; Y_{2}\right) & =h\left(S_{2}+U_{2, \epsilon}\right)-h\left(S_{2}+U_{2, \epsilon} \mid Y_{2}\right) \\
& =\frac{1}{2} \log \frac{\sigma_{S_{2}}^{2}+\sigma_{U_{2, \epsilon}}^{2}}{d_{2}(\gamma)+\sigma_{U_{2, \epsilon}}^{2}} .
\end{aligned}
$$

Therefore,

$$
\frac{\sigma_{S_{2}}^{2}+\sigma_{U_{2, \epsilon}}^{2}}{d_{2}(\gamma)+\sigma_{U_{2, \epsilon}}^{2}}=\frac{P(\gamma)+N_{2}}{\alpha_{\epsilon} P(\gamma)+N_{2}} .
$$

Note that

$$
\begin{aligned}
& I\left(\mathbf{S}_{1}+\mathbf{U}_{1, \epsilon} ; \mathbf{S}_{1}(\gamma), S_{2}(\gamma) \mid S_{2}+U_{2, \epsilon}\right) \\
&= I\left(\mathbf{S}_{1}+\mathbf{U}_{1, \epsilon}, S_{2}+U_{2, \epsilon} ; \mathbf{S}_{1}(\gamma), S_{2}(\gamma)\right) \\
&-I\left(S_{2}+U_{2, \epsilon} ; \mathbf{S}_{1}(\gamma), S_{2}(\gamma)\right) \\
&= I\left(\mathbf{S}_{1}+\mathbf{U}_{1, \epsilon}, S_{2}+U_{2, \epsilon} ; \mathbf{S}_{1}(\gamma), S_{2}(\gamma)\right) \\
&-I\left(S_{2}+U_{2, \epsilon} ; S_{2}(\gamma)\right) \\
&= I\left(\mathbf{S}_{1}+\mathbf{U}_{1, \epsilon}, S_{2}+U_{2, \epsilon} ; \mathbf{S}_{1}(\gamma), S_{2}(\gamma)\right)-I\left(S_{2}+U_{2, \epsilon} ; Y_{1}\right) \\
&= \frac{1}{2} \log \frac{\left|\Sigma_{\mathbf{S}_{1}, S_{2}}+\Sigma_{\mathbf{U}_{1, \epsilon}, U_{2, \epsilon}}\right|}{\left|\boldsymbol{\Theta}(\gamma)+\Sigma_{\mathbf{U}_{1, \epsilon}, U_{2, \epsilon}}\right|}-\frac{1}{2} \log \frac{P(\gamma)+N_{1}}{\alpha_{\epsilon} P(\gamma)+N_{1}},
\end{aligned}
$$

where (43) is due to (41). On the other hand,

$$
\begin{aligned}
I\left(\mathbf{S}_{1}+\right. & \left.\mathbf{U}_{1, \epsilon} ; \mathbf{S}_{1}(\gamma), S_{2}(\gamma) \mid S_{2}+U_{2, \epsilon}\right) \\
= & h\left(\mathbf{S}_{1}+\mathbf{U}_{1, \epsilon} \mid S_{2}+U_{2, \epsilon}\right) \\
& -h\left(\mathbf{S}+\mathbf{U}_{1, \epsilon} \mid S_{2}+U_{2, \epsilon}, \mathbf{S}_{1}(\gamma), S_{2}(\gamma)\right) \\
= & h\left(\mathbf{S}_{1}+\mathbf{U}_{1, \epsilon} \mid S_{2}+U_{2, \epsilon}\right)-h\left(\mathbf{S}+\mathbf{U}_{1, \epsilon} \mid \mathbf{S}_{1}(\gamma)\right) \\
= & \frac{1}{2} \log \frac{\mid \Sigma_{\mathbf{S}_{1}, S_{2}}+\Sigma_{\mathbf{U}_{1, \epsilon}, U_{2, \epsilon} \mid}}{\left.\left|\boldsymbol{\Theta}_{1}(\gamma)+\Sigma_{\mathbf{U}_{1, \epsilon} \mid}\right| \sigma_{S_{2}}^{2}+\sigma_{U_{2, \epsilon}}^{2}\right)},
\end{aligned}
$$

where (45) is due to (41). Combining (46) and (44) gives

$$
\begin{aligned}
\left|\Sigma_{\mathbf{S}_{1}, S_{2}}+\Sigma_{\mathbf{U}_{1, \epsilon}, U_{2, \epsilon}}\right| & \\
(\gamma)+\Sigma_{\mathbf{U}_{1, \epsilon}} \mid\left(\sigma_{S_{2}}^{2}\right. & \left.+\sigma_{U_{2, \epsilon}}^{2}\right) \\
& =\frac{\left|\Sigma_{\mathbf{S}_{1}, S_{2}}+\Sigma_{\mathbf{U}_{1, \epsilon}, U_{2, \epsilon}}\right|\left(\alpha_{\epsilon} P(\gamma)+N_{1}\right)}{\left|\boldsymbol{\Theta}(\gamma)+\Sigma_{\mathbf{U}_{1, \epsilon}, U_{2, \epsilon}}\right|\left(P(\gamma)+N_{1}\right)}
\end{aligned}
$$

which, together with (40) and (42), implies that

$$
\begin{aligned}
P(\gamma)= & \lim _{\epsilon \rightarrow 0} N_{1} \frac{\left|\Sigma_{\mathbf{S}_{1}, S_{2}}+\Sigma_{\mathbf{U}_{1, \epsilon}, U_{2, \epsilon}}\right|}{\left|\boldsymbol{\Theta}_{1}(\gamma)+\Sigma_{\mathbf{U}_{1, \epsilon}}\right|\left|d_{2}(\gamma)+\sigma_{U_{2, \epsilon}}^{2}\right|} \\
& +\left(N_{2}-N_{1}\right) \frac{\sigma_{S_{2}}^{2}+\sigma_{U_{2, \epsilon}}^{2}}{d_{2}(\gamma)+\sigma_{U_{2, \epsilon}}^{2}}-N_{2} .
\end{aligned}
$$

Note that

$$
\begin{aligned}
& \Lambda_{1}\left(\mathbf{D}_{1}-\boldsymbol{\Theta}_{1}(\gamma)\right)=\mathbf{0} \\
& \quad \Rightarrow \Xi_{1}^{T} \Lambda_{1}\left(\mathbf{D}_{1}-\boldsymbol{\Theta}_{1}(\gamma)\right) \Xi_{1}=\mathbf{0} \\
& \Rightarrow \Pi_{1} \Xi_{1}^{T}\left(\mathbf{D}_{1}-\boldsymbol{\Theta}_{1}(\gamma)\right) \Xi_{1}=\mathbf{0},
\end{aligned}
$$

which further implies that $\Xi_{1}^{T}\left(\mathbf{D}_{1}-\boldsymbol{\Theta}_{1}(\gamma)\right) \Xi_{1}$ is of the form $\operatorname{diag}\left(\mathbf{0}_{r \times r}, \mathbf{A}\right)$, where $\mathbf{0}_{r \times r}$ denotes an $r \times r$ all-zero matrix. Also note that $\Xi_{1}^{T} \Sigma_{\mathbf{U}_{1, \epsilon}} \Xi_{1}=\Pi_{1, \epsilon}^{-1}-\Xi_{1}^{T} \Theta_{1}(\gamma) \Xi_{1}$. Therefore,

$$
\begin{aligned}
\lim _{\epsilon \rightarrow 0} & \frac{\left|\boldsymbol{\Theta}_{1}(\gamma)+\Sigma_{\mathbf{U}_{1, \epsilon}}\right|}{\left|\mathbf{D}_{1}+\Sigma_{\mathbf{U}_{1, \epsilon}}\right|} \\
= & \lim _{\epsilon \rightarrow 0} \frac{\left|\Xi_{1}^{T} \boldsymbol{\Theta}_{1}(\gamma) \Xi_{1}+\Xi_{1}^{T} \Sigma_{\mathbf{U}_{1, \epsilon}} \Xi_{1}\right|}{\left|\Xi_{1}^{T} \mathbf{D}_{1} \Xi_{1}+\Xi_{1}^{T} \Sigma_{\mathbf{U}_{1, \epsilon}} \Xi_{1}\right|} \\
= & \lim _{\epsilon \rightarrow 0} \frac{\left|\Pi_{1, \epsilon}^{-1}\right|}{\left|\Pi_{1, \epsilon}^{-1}+\Xi_{1}^{T}\left(\mathbf{D}_{1}-\boldsymbol{\Theta}_{1}(\gamma)\right) \Xi_{1}\right|} \\
= & \lim _{\epsilon \rightarrow 0} \frac{\left|\Pi_{1, \epsilon}^{-1}\right|}{\left|\Pi_{1, \epsilon}^{-1}+\operatorname{diag}\left(\mathbf{0}_{r \times r}, \mathbf{A}\right)\right|} \\
= & 1 .
\end{aligned}
$$


Now one can readily prove (35) by combining (47) and (48). This completes the proof of Theorem 2 for the case $d_{2} \leq d_{2}\left(\sigma_{S_{2}}^{2}\right)$.

By restricting $\Sigma_{\mathbf{U}_{1}, U_{2}}$ to the form $\operatorname{diag}\left(\Sigma_{\mathbf{U}_{1}}, \sigma_{U_{2}}^{2}\right)$ and letting $\sigma_{U_{2}}^{2} \rightarrow \infty$, we can obtain the following lower bound from Corollary 1:

$$
P\left(\mathbf{D}_{1}, d_{2}\right) \geq \sup _{\Sigma_{\mathbf{U}_{1} \succ 0}} N_{1} \frac{\left|\Sigma_{\mathbf{S}_{1}}+\Sigma_{\mathbf{U}_{1}}\right|}{\left|\mathbf{D}_{1}+\Sigma_{\mathbf{U}_{1}}\right|}-N_{1} .
$$

Note that if $\gamma>\sigma_{S_{2}}^{2}$, then $\boldsymbol{\Theta}(\gamma)=\boldsymbol{\Theta}\left(\sigma_{S_{2}}^{2}\right)$ and $d_{2}(\gamma)=d_{2}\left(\sigma_{S_{2}}^{2}\right)$; moreover, in this case we have $\lambda_{2}=0$ (which implies that $\sigma_{U_{2, \epsilon}}^{2}$ tends to infinity as $\epsilon \rightarrow 0$ ), and consequently

$$
\begin{aligned}
P(\gamma)= & \lim _{\epsilon \rightarrow 0} N_{1} \frac{\left|\Sigma_{\mathbf{S}_{1}, S_{2}}+\Sigma_{\mathbf{U}_{1, \epsilon}, U_{2, \epsilon}}\right|}{\left|\mathbf{D}_{1}+\Sigma_{\mathbf{U}_{1, \epsilon}}\right|\left|d_{2}(\gamma)+\sigma_{U_{2, \epsilon}}^{2}\right|} \\
& +\left(N_{2}-N_{1}\right) \frac{\sigma_{S_{2}}^{2}+\sigma_{U_{2, \epsilon}}^{2}}{d_{2}(\gamma)+\sigma_{U_{2, \epsilon}}^{2}}-N_{2} \\
= & \lim _{\epsilon \rightarrow 0} N_{1} \frac{\left|\Sigma_{\mathbf{S}_{1}}+\Sigma_{\mathbf{U}_{1, \epsilon}}\right|}{\left|\mathbf{D}_{1}+\Sigma_{\mathbf{U}_{1, \epsilon}}\right|}-N_{1} .
\end{aligned}
$$

Therefore, the lower bound in (49) is tight when $d_{2}>d_{2}\left(\sigma_{S_{2}}^{2}\right)$, which completes the proof of Theorem 2 .

It is instructive to note that the role of $I\left(S_{2}+U_{2, \epsilon} ; Y_{2}\right)$ and $I\left(\mathbf{S}_{1}+\mathbf{U}_{1, \epsilon} ; \mathbf{S}_{1}(\gamma), S_{2}(\gamma) \mid S_{2}+U_{2, \epsilon}\right)$ in the achievability argument is similar to that of $I\left(\mathbf{V}_{2}^{n} ; Y_{2}^{n}\right)$ and $I\left(\mathbf{V}_{1}^{n} ; Y_{1}^{n} \mid \mathbf{V}_{2}^{n}\right)$ in the proof of Corollary 1. One can also readily see that (39) and (41) imply

$$
\begin{aligned}
& \lim _{\epsilon \rightarrow 0} I\left(\mathbf{S}_{1}+\mathbf{U}_{1, \epsilon}, S_{2}+U_{2, \epsilon} ; \mathbf{S}_{1}(\gamma), S_{2}(\gamma)\right) \\
& \quad=I\left(\mathbf{S}_{1}, S_{2} ; \mathbf{S}_{1}(\gamma), S_{2}(\gamma)\right), \\
& I\left(\mathbf{S}_{1}+\mathbf{U}_{1, \epsilon}, S_{2}+U_{2, \epsilon} \mid \mathbf{S}_{1}(\gamma), S_{2}(\gamma)\right)=0
\end{aligned}
$$

respectively. These two equations can be viewed as the counterparts of (23) and (24).

It is implicitly assumed in our construction that $\Sigma_{\mathbf{S}_{1}, S_{2}} \succ \mathbf{0}$, $\mathbf{D}_{1} \succ \mathbf{0}$, and $d_{2}>0$. In fact, Theorem 2 also holds in the degenerate case where the source covariance matrix and the distortions are not strictly positive definite, i.e., we can relax the condition to $\Sigma_{\mathbf{S}_{1}, S_{2}} \succeq \mathbf{0}$ (which includes the case where $S_{2}$ is a linear function of $\left.\mathbf{S}_{1}\right), \mathbf{D}_{1} \succeq \mathbf{0}$, and $d_{2} \geq 0$. It is straightforward to verify that Corollary 1 is directly applicable in this setup. For the achievability part, one can leverage the construction for the non-degenerate case via a simple perturbation argument. The details are left to the interested reader.

\section{B. Alternative Optimal Hybrid Schemes}

It turns out that in the vector-scalar case the hybrid scheme that achieves the optimal tradeoff between the transmit power and the reconstruction distortion pair is in general not unique. Specifically, we shall show that if the optimal solution to (30) is of the form ${ }^{4} \boldsymbol{\Theta}(\gamma)=\operatorname{diag}\left(\boldsymbol{\Theta}_{1}(\gamma), \theta_{2}(\gamma)\right)$, then there exists

\footnotetext{
${ }^{4}$ Note that this condition is satisfied if $\operatorname{diag}\left(\mathbf{D}_{1}, \gamma\right) \preceq \Sigma_{\mathbf{S}_{1}, S_{2}}$. In this case it follows by Fischer's inequality that $\boldsymbol{\Theta}(\gamma)=\operatorname{diag}\left(\mathbf{D}_{1}, \gamma\right)$.
}

a class of hybrid schemes with the same performance as that in Section IV-A.

Some additional notation needs to be introduced first. Recall $\mathbf{S}_{1}(\gamma), S_{2}(\gamma), \mathbf{Q}_{1}$, and $Q_{2}$ defined in Section IV-A, and define $\Delta=S_{2}(\gamma)-\mathbb{E}\left[S_{2}(\gamma) \mid \mathbf{S}_{1}(\gamma)\right]$. Now write $\Delta=\Delta_{0}+$ $\Delta_{1}+\Delta_{2}$, where $\Delta_{0}, \Delta_{1}$, and $\Delta_{2}$ are mutually independent zero-mean Gaussian random variables with variances to be specified. Furthermore, let $S_{0}(\gamma)=\mathbb{E}\left[S_{2}(\gamma) \mid \mathbf{S}_{1}(\gamma)\right]+\Delta_{0}$ and $S_{2}^{\prime}(\gamma)=S_{0}(\gamma)+\Delta_{1}$. Note that $\left(\mathbf{Q}_{1}, Q_{2}\right)$ is independent of $\left(\Delta_{0}, \Delta_{1}, \Delta_{2}\right)$; moreover, since $\boldsymbol{\Theta}(\gamma)=\operatorname{diag}\left(\boldsymbol{\Theta}_{1}(\gamma), \theta_{2}(\gamma)\right)$, it follows that $\mathbf{Q}_{1}$ and $Q_{2}$ are mutually independent. Therefore, $\mathbf{S}_{1} \leftrightarrow \mathbf{S}_{1}(\gamma) \leftrightarrow S_{0}(\gamma) \leftrightarrow S_{2}^{\prime}(\gamma) \leftrightarrow S_{2}$ form a Markov chain.

Note that

$$
\begin{aligned}
S_{0}(\gamma) & =\mathbb{E}\left[S_{0}(\gamma) \mid \mathbf{S}_{1}, S_{2}\right]+\bar{W}_{0} \\
& =\overline{\mathbf{a}}_{1}^{T} \mathbf{S}_{1}+\bar{a}_{2} S_{2}+\bar{W}_{0}, \\
\mathbf{S}_{1}(\gamma) & =\mathbb{E}\left[\mathbf{S}_{1}(\gamma) \mid \mathbf{S}_{1}, S_{0}(\gamma)\right]+\overline{\mathbf{W}}_{1} \\
& =\overline{\mathbf{B}}_{1} \mathbf{S}_{1}+\overline{\mathbf{b}}_{2} S_{0}(\gamma)+\overline{\mathbf{W}}_{1}, \\
S_{2}^{\prime}(\gamma) & =\mathbb{E}\left[S_{2}^{\prime}(\gamma) \mid S_{2}, S_{0}(\gamma)\right]+\bar{W}_{2} \\
& =\bar{c}_{1} S_{2}+\bar{c}_{2} S_{0}(\gamma)+\bar{W}_{2},
\end{aligned}
$$

where $\bar{W}_{0}$ is independent of $\left(\mathbf{S}_{1}, S_{2}\right), \overline{\mathbf{W}}_{1}$ is independent of $\left(\mathbf{S}_{1}, S_{2}, S_{0}(\gamma), S_{2}^{\prime}(\gamma)\right)$, and $\bar{W}_{2}$ is independent of $\left(\mathbf{S}_{1}, S_{2}, S_{0}(\gamma), \mathbf{S}_{1}(\gamma)\right)$. We define

$$
\begin{aligned}
\overline{\mathbf{S}}_{1}(\gamma) & =\overline{\mathbf{B}}_{1} \mathbf{S}_{1}+\overline{\mathbf{W}}_{1}, \\
\bar{S}_{2}(\gamma) & =\bar{c}_{1} S_{2}+\bar{W}_{2} .
\end{aligned}
$$

We are now in a position to describe the scheme (see Fig. 3).

1) Encoding: Let the channel input $X^{n}$, with average power $P(\gamma)$, be a superposition of an analog signal $X_{a}^{n}$ and two digital signals $X_{d, 1}^{n}$ and $X_{d, 2}^{n}$ (i.e., $X^{n}=X_{a}^{n}+X_{d, 1}^{n}+X_{d, 2}^{n}$ ). The analog portion is given by $X_{a}^{n}=\bar{\beta}\left(\overline{\mathbf{a}}_{1}^{T} \mathbf{S}_{1}^{n}+\bar{a}_{2} S_{2}^{n}\right)$ for some non-negative number $\bar{\beta}$ to be specified later. For the digital portion $X_{d, 2}^{n}$, the encoder first uses a Wyner-Ziv code of rate $R_{2}$ with codewords generated according to $\bar{S}_{2}(\gamma)$, with $S_{2}^{n}$ as the input, and with $X_{a}^{n}+X_{d, 1}^{n}+Z_{2}^{n}$ as the decoder side information; the encoder then determines $X_{d, 2}^{n}$ to send the bin index of the chosen Wyner-Ziv codeword $\bar{S}_{2}^{n}(\gamma)$ by using a channel code of rate $R_{2}$. For the digital portion $X_{d, 1}^{n}$, the encoder first uses a Wyner-Ziv code of rate $R_{1}$ with codewords generated according to $\overline{\mathbf{S}}_{1}(\gamma)$, with $\mathbf{S}_{1}^{n}$ as the input, and with $X_{a}^{n}+X_{d, 1}^{n}+Z_{1}^{n}$ as the decoder side information; the encoder then determines $X_{d, 1_{-}}^{n}$ to send the bin index of the chosen Wyner-Ziv codeword $\overline{\mathbf{S}}_{1}^{n}(\gamma)$ by using a dirty paper code of rate $R_{1}$ with $X_{a}^{n}$ treated as the channel state information known at the encoder. We define $P_{a}=\mathbb{E}\left[\left(X_{a}\right)^{2}\right]$ and $P_{d, i}=\mathbb{E}\left[\left(X_{d, i}\right)^{2}\right]$, $i=1,2$, where $X_{a} \triangleq \bar{\beta}\left(\overline{\mathbf{a}}_{1}^{T} \mathbf{S}_{1}+\bar{a}_{2} S_{2}\right), X_{d, 1}, X_{d, 2}$ are mutually independent zero-mean Gaussian random variables, and $P_{a}+P_{d, 1}+P_{d, 2}=P(\gamma)$.

2) Decoding: Receiver 2 decodes the channel code $X_{d, 2}^{n}$, subtracts it from the channel output $Y_{2}^{n} \triangleq X_{a}^{n}+X_{d, 1}^{n}+$ $X_{d, 2}^{n}+Z_{2}^{n}$, and recovers $\bar{S}_{2}^{n}(\gamma)$ by decoding the Wyner-Ziv code (the one of rate $R_{2}$ ) with $X_{a}^{n}+X_{d, 1}^{n}+Z_{2}^{n}$ as the side information. Furthermore, in view of the fact that the linear MMSE estimate of $S_{2}$ based on $\left(\bar{S}_{2}(\gamma), X_{a}+X_{d, 1}+Z_{2}\right)$ is $\hat{S}_{2}(\gamma) \triangleq \rho_{1} \bar{S}_{2}(\gamma)+\rho_{2}\left(X_{a}+X_{d, 1}+Z_{2}\right)$, where $\left(\rho_{1}, \rho_{2}\right)$ is an 

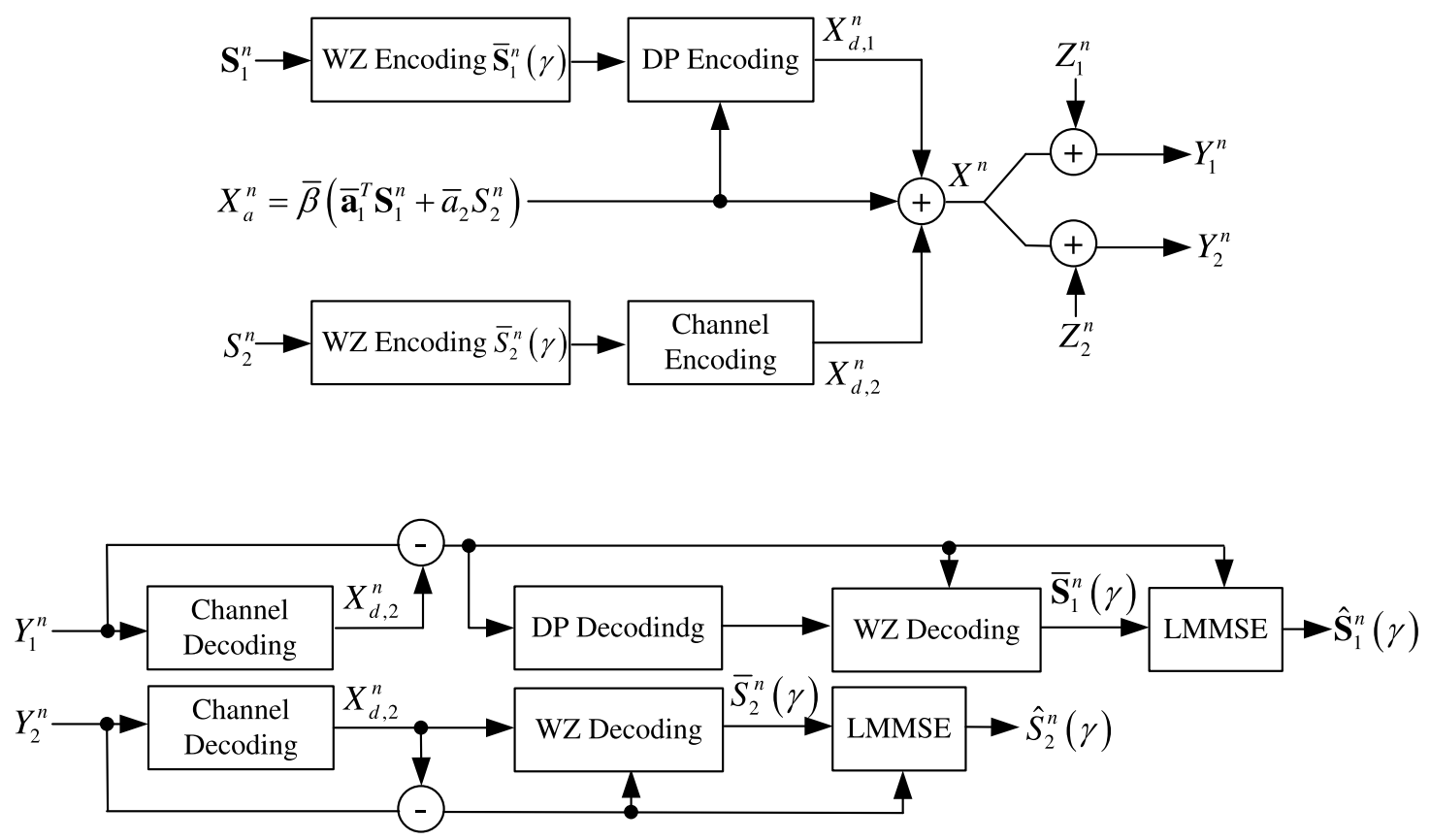

Fig. 3. An illustration of the hybrid scheme in Section IV-B.

arbitrary solution to the following equation

$$
\begin{aligned}
\left(\rho_{1}, \rho_{2}\right)\left(\begin{array}{cc}
\mathbb{E}\left[\left(\bar{S}_{2}(\gamma)\right)^{2}\right] & \mathbb{E}\left[\bar{S}_{2}(\gamma) X_{a}\right] \\
\mathbb{E}\left[\bar{S}_{2}(\gamma) X_{a}\right] & P_{a}+P_{d, 1}+N_{2}
\end{array}\right) \\
=\left(\mathbb{E}\left[S_{2} \bar{S}_{2}(\gamma)\right], \mathbb{E}\left[S_{2} X_{a}\right]\right),
\end{aligned}
$$

Receiver 2 can use $\hat{S}_{2}^{n}(\gamma) \triangleq \rho_{1} \bar{S}_{2}^{n}(\gamma)+\rho_{2}\left(X_{a}^{n}+X_{d, 1}^{n}+Z_{2}^{n}\right)$ as the reconstruction of $S_{2}^{n}$; the resulting distortion is denoted by $d_{2}(\gamma)$. Receiver 1 also decodes the channel code $X_{d, 2}^{n}$ and subtracts it from the channel output $Y_{1}^{n} \triangleq X_{a}^{n}+X_{d, 1}^{n}+$ $X_{d, 2}^{n}+Z_{1}^{n}$. Then Receiver 1 decodes the dirty paper code and recovers $\overline{\mathbf{S}}_{1}^{n}(\gamma)$ by decoding the Wyner-Ziv code (the one of rate $R_{1}$ ) with $X_{a}^{n}+X_{d, 1}^{n}+Z_{1}^{n}$ as the side information. Furthermore, in view of the fact that the linear MMSE estimate of $\mathbf{S}_{1}$ based on $\left(\overline{\mathbf{S}}_{1}(\gamma), X_{a}+X_{d, 1}+Z_{1}\right)$ is $\hat{\mathbf{S}}_{1}(\gamma) \triangleq \overline{\mathbf{S}}_{1}(\gamma)+$ $\bar{\beta}^{-1} \overline{\mathbf{b}}_{2}\left(X_{a}+X_{d, 1}+Z_{1}\right)$, Receiver 1 can use $\hat{\mathbf{S}}_{1}^{n}(\gamma) \triangleq \overline{\mathbf{S}}_{1}^{n}(\gamma)+$ $\bar{\beta}^{-1} \overline{\mathbf{b}}_{2}\left(X_{a}^{n}+X_{d, 1}^{n}+Z_{1}^{n}\right)$ as the reconstruction of $\mathbf{S}_{1}^{n}$.

3) Coding Parameters: Seven parameters $\mathbb{E}\left[\left(\Delta_{0}\right)^{2}\right]$, $\mathbb{E}\left[\left(\Delta_{1}\right)^{2}\right], \bar{\beta}, R_{1}, R_{2}, P_{d, 1}$, and $P_{d_{2}}$ still need to specified. Equivalently, we shall specify $\mathbb{E}\left[\left(\Delta_{0}\right)^{2}\right], \mathbb{E}\left[\left(\Delta_{1}\right)^{2}\right], P_{a}, R_{1}$, $R_{2}, P(\gamma)$, and $P_{d_{2}}$.

We again choose $P(\gamma)$ such that

$$
I\left(\mathbf{S}_{1}, S_{2} ; \mathbf{S}_{1}(\gamma), S_{2}(\gamma)\right)=\frac{1}{2} \log \frac{P(\gamma)+N_{1}}{N_{1}} .
$$

Let $P_{d, 2}$ be an arbitrary number in $\left[0, P_{d, 2}^{*}\right]$, where $P_{d, 2}^{*}$ is determined by the following equation

$$
\frac{1}{2} \log \frac{P(\gamma)-P_{d, 2}^{*}+N_{1}}{N_{1}}=I\left(\mathbf{S}_{1}, S_{2} ; \mathbf{S}_{1}(\gamma)\right) .
$$

Note that $P_{d, 2}^{*}$ is nonnegative since

$$
\begin{aligned}
I\left(\mathbf{S}_{1}, S_{2} ; \mathbf{S}_{1}(\gamma)\right) & \leq I\left(\mathbf{S}_{1}, S_{2} ; \mathbf{S}_{1}(\gamma), S_{2}(\gamma)\right) \\
& =\frac{1}{2} \log \frac{P(\gamma)+N_{1}}{N_{1}} .
\end{aligned}
$$

Now choose $\mathbb{E}\left[\left(\Delta_{0}\right)^{2}\right]$ such that

$$
I\left(\mathbf{S}_{1}, S_{2} ; \mathbf{S}_{1}(\gamma), S_{0}(\gamma)\right)=\frac{1}{2} \log \frac{P(\gamma)-P_{d, 2}+N_{1}}{N_{1}} .
$$

The existence of such $\mathbb{E}\left[\left(\Delta_{0}\right)^{2}\right]$ is guaranteed by the fact that one can let $I\left(\mathbf{S}_{1}, S_{2} ; \mathbf{S}_{1}(\gamma), S_{0}(\gamma)\right)$ take any value in $\left[I\left(\mathbf{S}_{1}, S_{2} ; \mathbf{S}_{1}(\gamma)\right), I\left(\mathbf{S}_{1}, S_{2} ; \mathbf{S}_{1}(\gamma), S_{2}(\gamma)\right)\right]$ (i.e., $\left.\left[\frac{1}{2} \log \frac{P(\gamma)-P_{d, 2}^{*}+N_{1}}{N_{1}}, \frac{1}{2} \log \frac{P(\gamma)+N_{1}}{N_{1}}\right]\right)$ by varying $\mathbb{E}\left[\left(\Delta_{0}\right)^{2}\right]$. We then choose $P_{a} \in\left[0, P(\gamma)-P_{d, 2}\right]$ (which further determines $P_{d, 1}$ and $\bar{\beta}$ ) such that

$$
I\left(X_{a} ; X_{a}+X_{d, 1}+Z_{1}\right)=I\left(\mathbf{S}_{1}, S_{2} ; S_{0}(\gamma)\right),
$$

which is always possible in view of (51) and the fact that one can let $I\left(X_{a} ; X_{a}+X_{d, 1}+Z_{1}\right)$ take any value in $\left[0, \frac{1}{2} \log \frac{P(\gamma)-P_{d, 2}+N_{1}}{N_{1}}\right]$ by varying $P_{a}$. Next we set

$$
R_{1}=I\left(\mathbf{S}_{1} ; \overline{\mathbf{S}}_{1}(\gamma) \mid X_{a}+X_{d, 1}+Z_{1}\right) .
$$

We finally choose $\mathbb{E}\left[\left(\Delta_{1}\right)^{2}\right]$ such that

$$
\begin{aligned}
I\left(S_{2} ; \bar{S}_{2}(\gamma) \mid X_{a}+X_{d, 1}\right. & \left.+Z_{2}\right) \\
& =\frac{1}{2} \log \frac{P(\gamma)+N_{2}}{P_{a}+P_{d, 1}+N_{2}}
\end{aligned}
$$

and set

$$
R_{2}=I\left(S_{2} ; \bar{S}_{2}(\gamma) \mid X_{a}+X_{d, 1}+Z_{2}\right) .
$$

It is not immediately clear that our particular choice of $\mathbb{E}\left[\left(\Delta_{1}\right)^{2}\right]$ always exists. To stress the dependence of $I\left(S_{2} ; \bar{S}_{2}(\gamma) \mid X_{a}+X_{d, 1}+Z_{2}\right)$ on $\mathbb{E}\left[\left(\Delta_{1}\right)^{2}\right]$, we shall denote it by $\psi\left(\mathbb{E}\left[\left(\Delta_{1}\right)^{2}\right]\right)$. Note that (52), together with the fact that $I\left(X_{a} ; X_{a}+X_{d, 1}+Z_{1}\right)=I\left(\mathbf{S}_{1}, S_{2} ; X_{a}+X_{d, 1}+Z_{1}\right)$, implies that $I\left(\mathbf{S}_{1}, S_{2} ; X_{a}+X_{d, 1}+Z_{1}\right)=I\left(\mathbf{S}_{1}, S_{2} ; S_{0}(\gamma)\right)$; moreover, since both $X_{d, 1}+Z_{1}$ and $\bar{W}_{0}$, which are Gaussian random variables, are independent of $\left(\mathbf{S}_{1}, S_{2}\right)$, it follows that 
the joint distributions of $\left(\mathbf{S}_{1}, S_{2}, \bar{\beta}^{-1}\left(X_{a}+X_{d, 1}+Z_{1}\right)\right)$ and $\left(\mathbf{S}_{1}, S_{2}, S_{0}(\gamma)\right)$ are identical, which, in view of the fact that $\overline{\mathbf{W}}_{1}$ and $\bar{W}_{2}$ are independent of $\left(\mathbf{S}_{1}, S_{2}, S_{0}(\gamma), X_{a}+X_{d, 1}+Z_{1}\right)$, further implies that the joint distributions of $\left(\mathbf{S}_{1}, S_{2}, \bar{\beta}^{-1}\left(X_{a}+\right.\right.$ $\left.\left.X_{d, 1}+Z_{1}\right), \overline{\mathbf{S}}_{1}(\gamma), \bar{S}_{2}(\gamma)\right)$ and $\left(\mathbf{S}_{1}, S_{2}, S_{0}(\gamma), \overline{\mathbf{S}}_{1}(\gamma), \bar{S}_{2}(\gamma)\right)$ are identical..$^{5}$ Therefore, we have

$$
\begin{aligned}
& \psi\left(\mathbb{E}\left[\left(\Delta_{1}\right)^{2}\right]\right) \\
&= I\left(X_{a}+X_{d, 1}+Z_{2}, S_{2} ; \bar{S}_{2}(\gamma)\right) \\
&-I\left(X_{a}+X_{d, 1}+Z_{2} ; \bar{S}_{2}(\gamma)\right) \\
&= I\left(S_{2} ; \bar{S}_{2}(\gamma)\right)-I\left(X_{a}+X_{d, 1}+Z_{2} ; \bar{S}_{2}(\gamma)\right) \\
& \geq I\left(S_{2} ; \bar{S}_{2}(\gamma)\right)-I\left(X_{a}+X_{d, 1}+Z_{1} ; \bar{S}_{2}(\gamma)\right) \\
&= I\left(S_{2} ; \bar{S}_{2}(\gamma)\right)-I\left(S_{0}(\gamma) ; \bar{S}_{2}(\gamma)\right) \\
&= I\left(S_{2} ; \bar{S}_{2}(\gamma) \mid S_{0}(\gamma)\right) \\
&= I\left(S_{2} ; S_{2}^{\prime}(\gamma) \mid S_{0}(\gamma)\right),
\end{aligned}
$$

where (56) is due to the fact that $\left(X_{a}+X_{d, 1}+Z_{2}\right) \leftrightarrow$ $S_{2} \leftrightarrow \bar{S}_{2}(\gamma)$ form a Markov chain. Clearly, $\psi\left(\mathbb{E}\left[\left(\Delta_{1}\right)^{2}\right]\right)$ is a continuous function of $\mathbb{E}\left[\left(\Delta_{1}\right)^{2}\right]$. When $\mathbb{E}\left[\left(\Delta_{1}\right)^{2}\right]=0$, we have $S_{2}^{\prime}(\gamma)=S_{0}(\gamma)$ (which implies $\bar{S}_{2}(\gamma)=0$ ) and consequently $\psi(0)=0$; when $\mathbb{E}\left[\left(\Delta_{1}\right)^{2}\right]=\mathbb{E}\left[(\Delta)^{2}\right]-\mathbb{E}\left[\left(\Delta_{0}\right)^{2}\right]$, we have $S_{2}^{\prime}(\gamma)=S_{2}(\gamma)$ and consequently $\psi\left(\mathbb{E}\left[(\Delta)^{2}\right]-\right.$ $\left.\mathbb{E}\left[\left(\Delta_{0}\right)^{2}\right]\right) \geq I\left(S_{2} ; S_{2}(\gamma) \mid S_{0}(\gamma)\right)$. Note that

$$
\begin{aligned}
\frac{1}{2} \log & \frac{P(\gamma)+N_{1}}{N_{1}} \\
& =I\left(\mathbf{S}_{1}, S_{2} ; \mathbf{S}_{1}(\gamma), S_{2}(\gamma)\right) \\
& =I\left(\mathbf{S}_{1}, S_{2} ; \mathbf{S}_{1}(\gamma), S_{2}(\gamma), S_{0}(\gamma)\right) \\
& =I\left(\mathbf{S}_{1}, S_{2} ; \mathbf{S}_{1}(\gamma), S_{0}(\gamma)\right)+I\left(S_{2} ; S_{2}(\gamma) \mid S_{0}(\gamma)\right) \\
& =\frac{1}{2} \log \frac{P_{a}+P_{d, 1}+N_{1}}{N_{1}}+I\left(S_{2} ; S_{2}(\gamma) \mid S_{0}(\gamma)\right),
\end{aligned}
$$

where (57) and (58) are due to (50) and (51), respectively. This implies

$$
I\left(S_{2} ; S_{2}(\gamma) \mid S_{0}(\gamma)\right)=\frac{1}{2} \log \frac{P(\gamma)+N_{1}}{P_{a}+P_{d, 1}+N_{1}} .
$$

Therefore, we have

$$
\begin{aligned}
\psi\left(\mathbb{E}\left[\Delta^{2}\right]-\mathbb{E}\left[\Delta_{0}^{2}\right]\right) & \geq \frac{1}{2} \log \frac{P(\gamma)+N_{1}}{P_{a}+P_{d, 1}+N_{1}} \\
& \geq \frac{1}{2} \log \frac{P(\gamma)+N_{2}}{P_{a}+P_{d, 1}+N_{2}} .
\end{aligned}
$$

Hence, our choice of $\mathbb{E}\left[\Delta_{1}^{2}\right]$ indeed exists.

4) Conditions for Correct Decoding: Receiver 2 needs to decode the channel code and the corresponding Wyner-Ziv code of rate $R_{2}$, and the correct decoding of these two components are guaranteed by (54) and (55). Since Receiver 1 is stronger than Receiver 2, it can also decode the channel code and subtract it from the channel output. Receiver 1 additionally needs to decode the dirty paper code and the corresponding Wyner-Ziv code of rate $R_{1}$, the latter of which is guaranteed by (53).

\footnotetext{
${ }^{5}$ We have implicitly assumed that $\mathbb{E}\left[\left(\overline{\mathbf{a}}_{1}^{T} \mathbf{S}_{1}+\bar{a}_{2} S_{2}\right)^{2}\right]>0$ (which implies that the $P_{a}$ and the $\bar{\beta}$ determined by (52) are positive). For the degenerate case $\overline{\mathbf{a}}_{1}^{T} \mathbf{S}_{1}+\bar{a}_{2} S_{2}=0$ (which is possible if and only if $S_{0}(\gamma)=0$ ), one can simply set $X_{a}=0$ and $\bar{\beta}^{-1}\left(X_{a}+X_{d, 1}+Z_{1}\right)=0$.
}

Recall that the joint distributions of $\left(\mathbf{S}_{1}, S_{2}, \bar{\beta}^{-1}\left(X_{a}+\right.\right.$ $\left.\left.X_{d, 1}+Z_{1}\right), \overline{\mathbf{S}}_{1}(\gamma), \bar{S}_{2}(\gamma)\right)$ and $\left(\mathbf{S}_{1}, S_{2}, S_{0}(\gamma), \overline{\mathbf{S}}_{1}(\gamma), \bar{S}_{2}(\gamma)\right)$ are identical. Therefore, we have

$$
\begin{aligned}
R_{1} & =I\left(\mathbf{S}_{1} ; \overline{\mathbf{S}}_{1}(\gamma) \mid X_{a}+X_{d, 1}+Z_{1}\right) \\
& =I\left(\mathbf{S}_{1} ; \mathbf{S}_{1}(\gamma) \mid S_{0}(\gamma)\right) \\
& =I\left(\mathbf{S}_{1}, S_{2} ; \mathbf{S}_{1}(\gamma) \mid S_{0}(\gamma)\right) \\
& =I\left(\mathbf{S}_{1}, S_{2} ; \mathbf{S}_{1}(\gamma), S_{0}(\gamma)\right)-I\left(\mathbf{S}_{1}, S_{2} ; S_{0}(\gamma)\right) \\
& =\frac{1}{2} \log \frac{P_{a}+P_{d, 1}+N_{1}}{N_{1}}-\frac{1}{2} \log \frac{P_{a}+P_{d, 1}+N_{1}}{P_{d, 1}+N_{1}} \\
& =\frac{1}{2} \log \frac{P_{d, 1}+N_{1}}{N_{1}},
\end{aligned}
$$

where (59) follows by the fact that $S_{2} \leftrightarrow\left(S_{0}(\gamma), \mathbf{S}_{1}\right) \leftrightarrow \mathbf{S}_{1}(\gamma)$ form a Markov chain (which is implied by the fact that $S_{2}-S_{0}(\gamma) \leftrightarrow \mathbf{S}_{1}(\gamma) \leftrightarrow \mathbf{S}_{1}$ form a Markov chain), and (60) is due to (51) and (52). Thus indeed Receiver 1 can decode the dirty paper code correctly.

5) Optimality of This Class of Schemes: Since the joint distributions of $\left(\mathbf{S}_{1}, \hat{\mathbf{S}}_{1}(\gamma)\right)$ and $\left(\mathbf{S}_{1}, \mathbf{S}_{1}(\gamma)\right)$ are identical (which is due to the fact that the joint distributions of $\left(\mathbf{S}_{1}, \bar{\beta}^{-1}\left(X_{a}+\right.\right.$ $\left.\left.X_{d, 1}+Z_{1}\right), \overline{\mathbf{S}}_{1}(\gamma)\right)$ and $\left(\mathbf{S}_{1}, S_{0}(\gamma), \overline{\mathbf{S}}_{1}(\gamma)\right)$ are identical), it follows that the resulting distortion at Receiver 1 is $\boldsymbol{\Theta}_{1}(\gamma)$, which is the same as that achieved by the optimal scheme given in Section IV-A. We next focus on the distortion achieved at Receiver 2.

Note that we have the freedom to choose $P_{d, 2}$ from $\left[0, P_{d, 2}^{*}\right]$. In particular, one can recover the hybrid scheme in Section IV-A by setting $P_{d, 2}=0$. We shall show ${ }^{6}$ that the reconstruction distortion at Receiver 2 (i.e., $d_{2}(\gamma)$ ) does not depend on $P_{d, 2}$; as a consequence, this class of schemes have exactly the same performance, and can all achieve the optimal tradeoff between the transmit power and the reconstruction distortion pair. Note that

$$
\begin{aligned}
& \frac{1}{2} \log \frac{\left|\mathbb{E}\left[\left(\mathbf{S}_{1}-\mathbb{E}\left[\mathbf{S}_{1} \mid S_{2}\right]\right)\left(\mathbf{S}_{1}-\mathbb{E}\left[\mathbf{S}_{1} \mid S_{2}\right]\right)^{T}\right]\right|}{\left|\mathbf{\Theta}_{1}(\gamma)\right|} \\
&= h\left(\mathbf{S}_{1} \mid S_{2}\right)-h\left(\mathbf{S}_{1} \mid \mathbf{S}_{1}(\gamma)\right) \\
&= h\left(\mathbf{S}_{1} \mid S_{2}\right)-h\left(\mathbf{S}_{1} \mid S_{2}, S_{0}(\gamma), \mathbf{S}_{1}(\gamma)\right) \\
&= I\left(\mathbf{S}_{1} ; S_{0}(\gamma), \mathbf{S}_{1}(\gamma) \mid S_{2}\right) \\
&= I\left(\mathbf{S}_{1} ; X_{a}+X_{d, 1}+Z_{1}, \hat{\mathbf{S}}_{1}(\gamma) \mid S_{2}\right) \\
&= I\left(\mathbf{S}_{1} ; X_{a}+X_{d, 1}+Z_{1}, \overline{\mathbf{S}}_{1}(\gamma) \mid S_{2}\right) \\
&= I\left(\mathbf{S}_{1} ; X_{a}+X_{d, 1}+Z_{1} \mid S_{2}\right) \\
&+I\left(\mathbf{S}_{1} ; \overline{\mathbf{S}}_{1}(\gamma) \mid X_{a}+X_{d, 1}+Z_{1}\right) \\
&= I\left(\mathbf{S}_{1} ; X_{a}+X_{d, 1}+Z_{1} \mid S_{2}\right)+\frac{1}{2} \log \frac{P_{d, 1}+N_{1}}{N_{1}} \\
&= \frac{1}{2} \log \frac{\mathbb{E}\left[\left(X_{a}-\mathbb{E}\left[X_{a} \mid S_{2}\right]\right)^{2}\right]+P_{d, 1}+N_{1}}{P_{d, 1}+N_{1}} \\
&+\frac{1}{2} \log \frac{P_{d, 1}+N_{1}}{N_{1}} \\
&= \frac{1}{2} \log \frac{\mathbb{E}\left[\left(X_{a}-\mathbb{E}\left[X_{a} \mid S_{2}\right]\right)^{2}\right]+P_{d, 1}+N_{1}}{N_{1}},
\end{aligned}
$$

\footnotetext{
${ }^{6}$ It is clear that the reconstruction distortion at Receiver 1 (i.e., $\boldsymbol{\Theta}_{1}(\gamma)$ ) does not depend on $P_{d, 2}$.
} 

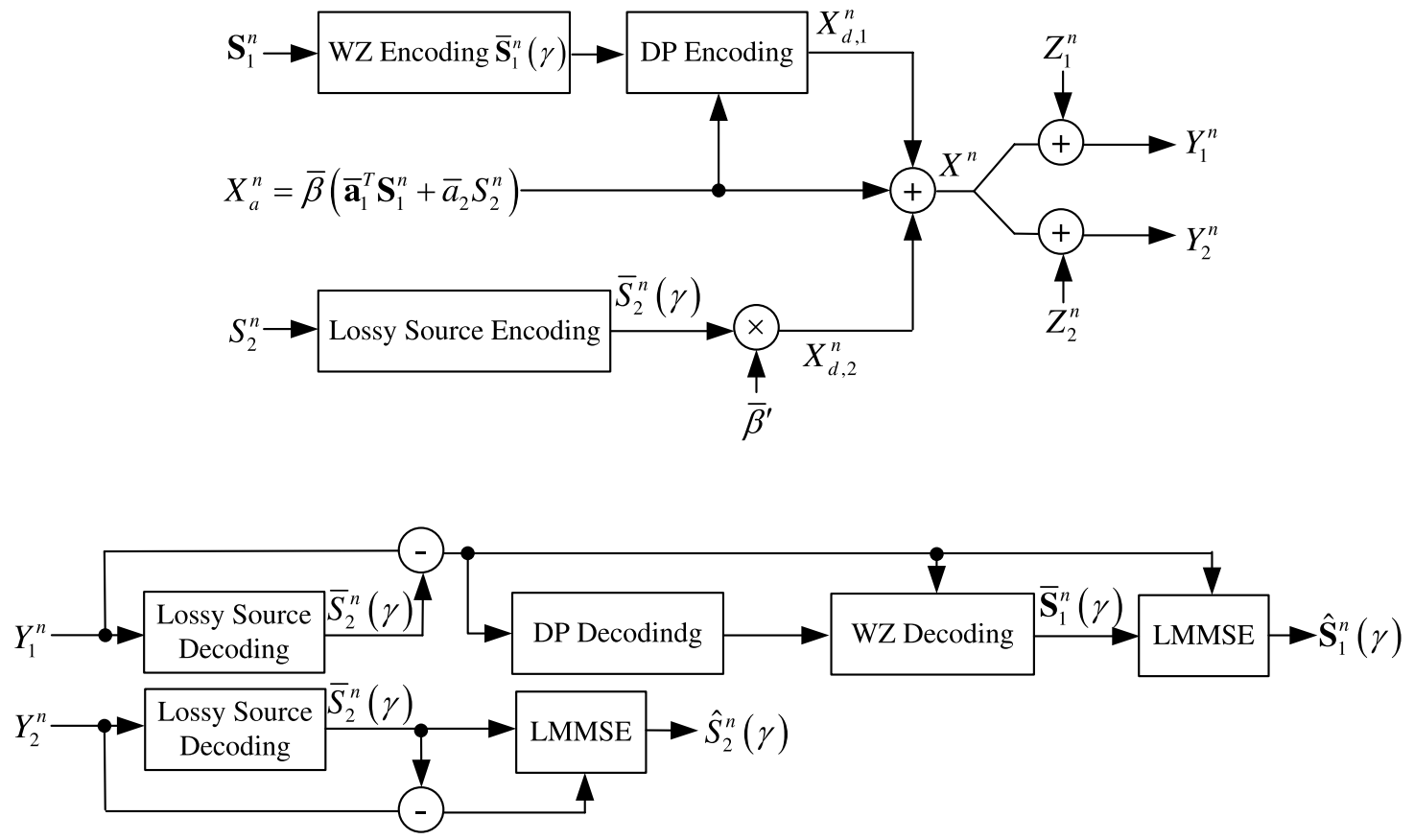

Fig. 4. A variant of the hybrid scheme in Section IV-B.

where (62) follows from the fact that $\mathbf{S}_{1} \leftrightarrow \mathbf{S}_{1}(\gamma) \leftrightarrow$ $\left(S_{2}, S_{0}(\gamma)\right)$ form a Markov chain, (63) follows from the fact that the joint distributions of $\left(\mathbf{S}_{1}, S_{2}, \bar{\beta}^{-1}\left(X_{a}+X_{d, 1}+Z_{1}\right)\right.$, $\left.\hat{\mathbf{S}}_{1}(\gamma)\right)$ and $\left(\mathbf{S}_{1}, S_{2}, S_{0}(\gamma), \mathbf{S}_{1}(\gamma)\right)$ are identical, and (64) is due to (61). Therefore, $\mathbb{E}\left[\left(X_{a}-\mathbb{E}\left[X_{a} \mid S_{2}\right]\right)^{2}\right]+P_{d, 1}$ is not affected by the choice of $P_{d, 2}$. Since

$$
\begin{aligned}
\frac{1}{2} \log \frac{\sigma_{S_{2}}^{2}}{d_{2}(\gamma)} \\
=I\left(S_{2} ; \bar{S}_{2}(\gamma), X_{a}+X_{d, 1}+Z_{2}\right) \\
=I\left(S_{2} ; X_{a}+X_{d, 1}+Z_{2}\right)+I\left(S_{2} ; \bar{S}_{2}(\gamma) \mid X_{a}+X_{d, 1}+Z_{2}\right) \\
=I\left(S_{2} ; X_{a}+X_{d, 1}+Z_{2}\right)+\frac{1}{2} \log \frac{P(\gamma)+N_{2}}{P_{a}+P_{d, 1}+N_{2}} \\
=\frac{1}{2} \log \frac{P_{a}+P_{d, 1}+N_{2}}{\mathbb{E}\left[\left(X_{a}-\mathbb{E}\left[X_{a} \mid S_{2}\right]\right)^{2}\right]+P_{d, 1}+N_{2}} \\
\quad+\frac{1}{2} \log \frac{P(\gamma)+N_{2}}{P_{a}+P_{d, 1}+N_{2}} \\
=\frac{1}{2} \log \frac{P(\gamma)+N_{2}}{\mathbb{E}\left[\left(X_{a}-\mathbb{E}\left[X_{a} \mid S_{2}\right]\right)^{2}\right]+P_{d, 1}+N_{2}}
\end{aligned}
$$

where (65) is due to (54), it follows that $d_{2}(\gamma)$ does not depend on $P_{d, 2}$.

6) A Variant of This Class of Optimal Schemes: For each $P_{d, 2} \in\left[0, P_{d, 2}^{*}\right]$, the aforedescribed scheme has the following variant (see Fig. 4). Now for the digital portion $X_{d, 2}^{n}$, the encoder simply uses a lossy source code of rate $I\left(S_{2} ; \bar{S}_{2}(\gamma)\right)$ with codewords generated according to $\bar{S}_{2}(\gamma)$ and with $S_{2}^{n}$ as the input, and sets $X_{d, 2}^{n}$ to be the output codeword $\bar{S}_{2}^{n}(\gamma)$ multiplied by some non-negative number $\bar{\beta}^{\prime}$, where $\bar{\beta}^{\prime}$ is chosen such that $\mathbb{E}\left[\left(X_{a}+X_{d, 1}+\bar{\beta}^{\prime} \bar{S}_{2}(\gamma)\right)^{2}\right]=P(\gamma)$. The remaining part of the encoder is still the same.
Define $Y_{i}=X_{a}+X_{d, 1}+\bar{\beta}^{\prime} \bar{S}_{2}(\gamma)+Z_{i}, i=1,2$. Note that

$$
\begin{aligned}
I & \left(S_{2} ; \bar{S}_{2}(\gamma)\right)+I\left(S_{2} ; X_{a}+X_{d, 1}+Z_{2} \mid \bar{S}_{2}(\gamma)\right) \\
& =I\left(S_{2} ; \bar{S}_{2}(\gamma), X_{a}+X_{d, 1}+Z_{2}\right) \\
& =\frac{1}{2} \log \frac{\sigma_{S_{2}}^{2}}{d_{2}(\gamma)} \\
& =\frac{1}{2} \log \frac{P(\gamma)+N_{2}}{\mathbb{E}\left[\left(X_{a}-\mathbb{E}\left[X_{a} \mid S_{2}\right]\right)^{2}\right]+P_{d, 1}+N_{2}} \\
& =h\left(Y_{2}\right)-h\left(Y_{2} \mid S_{2}, \bar{S}_{2}(\gamma)\right) \\
& =I\left(S_{2}, \bar{S}_{2}(\gamma) ; Y_{2}\right) \\
& =I\left(\bar{S}_{2}(\gamma) ; Y_{2}\right)+I\left(S_{2} ; Y_{2} \mid \bar{S}_{2}(\gamma)\right) \\
& =I\left(\bar{S}_{2}(\gamma) ; Y_{2}\right)+I\left(S_{2} ; X_{a}+X_{d, 1}+Z_{2} \mid \bar{S}_{2}(\gamma)\right),
\end{aligned}
$$

where (67) is due to (66). This implies

$$
I\left(S_{2} ; \bar{S}_{2}(\gamma)\right)=I\left(\bar{S}_{2}(\gamma) ; Y_{2}\right) .
$$

Hence, Receiver 2 can decode the lossy source code and recover $\bar{S}_{2}^{n}(\gamma)$. Furthermore, Receiver $2 \operatorname{can}^{7}$ use $\hat{S}_{2}^{n}(\gamma)$ as the reconstruction of $S_{2}^{n}$, and the resulting distortion is $d_{2}(\gamma)$. Receiver 1 can also decode the lossy source code and obtain $X_{a}^{n}+X_{d, 1}^{n}+Z_{1}^{n}$ based on $\bar{S}_{2}^{n}(\gamma)$ and $Y_{1}^{n} \triangleq X_{a}^{n}+X_{d, 1}^{n}+$ $\bar{\beta}^{\prime} \bar{S}_{2}^{n}(\gamma)+Z_{1}^{n}$. Then Receiver 1 decodes the dirty paper code and recovers $\overline{\mathbf{S}}_{1}^{n}(\gamma)$ by decoding the Wyner-Ziv code (the one of rate $R_{1}$ ) with $X_{a}^{n}+X_{d, 1}^{n}+Z_{1}^{n}$ as the side information. Moreover, Receiver 1 can use $\hat{\mathbf{S}}_{1}^{n}(\gamma)$ as the reconstruction of $\mathbf{S}_{1}^{n}$, and the resulting distortion is $\boldsymbol{\Theta}_{1}(\gamma)$. Therefore, this scheme has exactly the same performance as the original one. It is worth mentioning that the scheme in [10] can be viewed as an extremal case of this scheme with $P_{d, 2}=P_{d, 2}^{*}$ and $m_{1}=1$.

\footnotetext{
${ }^{7}$ Note that Receiver 2 can obtain $X_{a}^{n}+X_{d, 1}^{n}+Z_{2}^{n}$ from $\bar{S}_{2}^{n}(\gamma)$ and $Y_{2}^{n} \triangleq X_{a}^{n}+X_{d, 1}^{n}+\bar{\beta}^{\prime} \bar{S}_{2}^{n}(\gamma)+Z_{2}^{n}$.
} 


\section{CONClusion}

We have obtained a lower bound on the optimal tradeoff between the transmit power and the achievable distortion pair for the problem of sending correlated vector Gaussian sources over a Gaussian broadcast channel, where each receiver wishes to reconstruct its target source under a covariance distortion constraint. This lower bound is shown to be achievable by a class of hybrid schemes for the vector-scalar case, i.e., the scenario where the weak receiver wishes to reconstruct a scalar source under the mean squared error distortion constraint. For certain classes of sources and distortion matrices, it is possible to extend our hybrid schemes to obtain a characterization of the optimal power-distortion tradeoff for the case where the weak receiver also wishes to reconstruct a vector source. However, a complete solution for this general setup remains elusive.

\section{APPENDIX A}

\section{PROOF OF LEMMA 1}

Let $\mathbf{W}(t)$ and $\hat{\mathbf{W}}(t)$ be the $t$-th columns of $\mathbf{W}$ and $\hat{\mathbf{W}}$, respectively, $t=1, \ldots, n$. Note that

$$
\begin{aligned}
h(\mathbf{W} \mid \hat{\mathbf{W}}) \\
\quad=h(\mathbf{W}(1) \mid \hat{\mathbf{W}})+\sum_{t=2}^{n} h(\mathbf{W}(t) \mid \hat{\mathbf{W}}, \mathbf{W}(1), \ldots, \mathbf{W}(t-1)) \\
\quad \leq \sum_{t=1}^{n} h(\mathbf{W}(t) \mid \hat{\mathbf{W}}(t)) \\
\quad \leq \sum_{t=1}^{n} h(\mathbf{W}(t)-\hat{\mathbf{W}}(t)) \\
\quad \leq \sum_{t=1}^{n} \frac{1}{2} \log \left|2 \pi e \mathbb{E}\left[(\mathbf{W}(t)-\hat{\mathbf{W}}(t))(\mathbf{W}(t)-\hat{\mathbf{W}}(t))^{T}\right]\right| \\
\quad \leq \frac{n}{2} \log \left|\frac{2 \pi e}{n} \sum_{t=1}^{n} \mathbb{E}\left[(\mathbf{W}(t)-\hat{\mathbf{W}}(t))(\mathbf{W}(t)-\hat{\mathbf{W}}(t))^{T}\right]\right| \\
\quad=\frac{n}{2} \log \left|\frac{2 \pi e}{n} \mathbb{E}\left[(\mathbf{W}-\hat{\mathbf{W}})(\mathbf{W}-\hat{\mathbf{W}})^{T}\right]\right|,
\end{aligned}
$$

which completes the proof of Lemma 1.

\section{APPENDIX B}

\section{PROOF OF LEMMA 2}

Let $\mathbf{W}_{1}(t)$ and $\mathbf{W}_{2}(t)$ be the $t$-th columns of $\mathbf{W}_{1}$ and $\mathbf{W}_{2}$, respectively, $t=1, \ldots, n$. Let $\Gamma$ be uniformly distributed over $\{1, \ldots, n\}$ and independent of $\left(\mathbf{W}_{1}, \mathbf{W}_{2}\right)$. We have

$$
\begin{aligned}
h\left(\mathbf{W}_{1} \mid \mathbf{W}_{2}\right)= & h\left(\mathbf{W}_{1}(1) \mid \mathbf{W}_{2}\right) \\
& +\sum_{t=2}^{n} h\left(\mathbf{W}_{1}(t) \mid \mathbf{W}_{2}, \mathbf{W}_{1}(1), \ldots, \mathbf{W}_{1}(t-1)\right) \\
\leq & \sum_{t=1}^{n} h\left(\mathbf{W}_{1}(t) \mid \mathbf{W}_{2}(t)\right) \\
= & n h\left(\mathbf{W}_{1}(\Gamma) \mid \mathbf{W}_{2}(\Gamma), \Gamma\right) \\
\leq & n h\left(\mathbf{W}_{1}(\Gamma) \mid \mathbf{W}_{2}(\Gamma)\right) .
\end{aligned}
$$

It is easy to see that

$$
\begin{aligned}
\mathbb{E}\left[\left(\mathbf{W}_{1}^{T}(\Gamma), \mathbf{W}_{2}^{T}(\Gamma)\right)^{T}\left(\mathbf{W}_{1}^{T}(\Gamma), \mathbf{W}_{2}^{T}(\Gamma)\right)\right] \\
\quad=\frac{1}{n} \mathbb{E}\left[\left(\mathbf{W}_{1}^{T}, \mathbf{W}_{2}^{T}\right)^{T}\left(\mathbf{W}_{1}^{T}, \mathbf{W}_{2}^{T}\right)\right] .
\end{aligned}
$$

Let $\hat{\mathbf{W}}_{1}(\Gamma)$ be the linear MMSE estimate of $\mathbf{W}_{1}(\Gamma)$ based on $\mathbf{W}_{2}(\Gamma)$. Note that

$$
\begin{aligned}
& \left|\mathbb{E}\left(\left(\mathbf{W}_{1}(\Gamma)-\hat{\mathbf{W}}_{1}(\Gamma)\right)\left(\mathbf{W}_{1}(\Gamma)-\hat{\mathbf{W}}_{1}(\Gamma)\right)^{T}\right)\right| \\
& \quad=\frac{\left|\mathbb{E}\left[\left(\mathbf{W}_{1}^{T}(\Gamma), \mathbf{W}_{2}^{T}(\Gamma)\right)^{T}\left(\mathbf{W}_{1}^{T}(\Gamma), \mathbf{W}_{2}^{T}(\Gamma)\right)\right]\right|}{\left|\mathbb{E}\left[\mathbf{W}_{2}(\Gamma) \mathbf{W}_{2}^{T}(\Gamma)\right]\right|} \\
& \quad=\frac{\left|\frac{1}{n} \mathbb{E}\left[\left(\mathbf{W}_{1}^{T}, \mathbf{W}_{2}^{T}\right)^{T}\left(\mathbf{W}_{1}^{T}, \mathbf{W}_{2}^{T}\right)\right]\right|}{\left|\frac{1}{n} \mathbb{E}\left[\mathbf{W}_{2} \mathbf{W}_{2}^{T}\right]\right|} .
\end{aligned}
$$

Now continuing from (68),

$$
\begin{aligned}
n h & \left(\mathbf{W}_{1}(\Gamma) \mid \mathbf{W}_{2}(\Gamma)\right) \\
& \leq n h\left(\mathbf{W}_{1}(\Gamma)-\hat{\mathbf{W}}_{1}(\Gamma)\right) \\
& \leq \frac{n}{2} \log \left|2 \pi e \mathbb{E}\left(\left(\mathbf{W}_{1}(\Gamma)-\hat{\mathbf{W}}_{1}(\Gamma)\right)\left(\mathbf{W}_{1}(\Gamma)-\hat{\mathbf{W}}_{1}(\Gamma)\right)^{T}\right)\right| \\
& =\frac{n}{2} \log \frac{\left|\frac{2 \pi e}{n} \mathbb{E}\left[\left(\mathbf{W}_{1}^{T}, \mathbf{W}_{2}^{T}\right)^{T}\left(\mathbf{W}_{1}^{T}, \mathbf{W}_{2}^{T}\right)\right]\right|}{\left|\frac{2 \pi e}{n} \mathbb{E}\left[\mathbf{W}_{2} \mathbf{W}_{2}^{T}\right]\right|}
\end{aligned}
$$

where (70) is due to (69). This completes the proof of Lemma 2.

\section{APPENDIX C}

The Continuity of $\boldsymbol{\Theta}(\gamma)$

If $\boldsymbol{\Theta}(\gamma)$ is not continuous at $\gamma=\gamma^{*}$ for some $\gamma^{*}>0$, then there exists a sequence $\left\{\boldsymbol{\Theta}\left(\gamma_{k}\right)\right\}$ with $\gamma_{k} \rightarrow \gamma^{*}$ and $\boldsymbol{\Theta}\left(\gamma_{k}\right) \rightarrow \boldsymbol{\Theta}^{\prime}\left(\gamma^{*}\right) \neq \boldsymbol{\Theta}\left(\gamma^{*}\right)$ as $k \rightarrow \infty$. Clearly, $\boldsymbol{\Theta}^{\prime}\left(\gamma^{*}\right)$ satisfies the constraints for the maximization problem (with $\gamma=\gamma^{*}$ ) in (30). Therefore, we must have $\log \left|\boldsymbol{\Theta}^{\prime}\left(\gamma^{*}\right)\right| \leq \log \left|\boldsymbol{\Theta}\left(\gamma^{*}\right)\right|$. Now let $\tilde{\boldsymbol{\Theta}}\left(\gamma_{k}\right)=\boldsymbol{\Theta}\left(\gamma^{*}\right)-$ $\operatorname{diag}\left(\mathbf{0}, \max \left(\gamma^{*}-\gamma_{k}, 0\right)\right)$. Note that $\tilde{\boldsymbol{\Theta}}\left(\gamma_{k}\right)$ satisfies the constraints for the maximization problem (with $\gamma=\gamma_{k}$ ) in (30) when $\gamma_{k}$ is sufficiently close to $\gamma^{*}$. Therefore,

$$
\limsup _{k \rightarrow \infty} \log \left|\tilde{\boldsymbol{\Theta}}\left(\gamma_{k}\right)\right| \leq \lim _{k \rightarrow \infty} \log \left|\boldsymbol{\Theta}\left(\gamma_{k}\right)\right|=\log \left|\boldsymbol{\Theta}^{\prime}\left(\gamma^{*}\right)\right| .
$$

On the other hand, it is clear that

$$
\lim _{k \rightarrow \infty} \log \left|\tilde{\boldsymbol{\Theta}}\left(\gamma_{k}\right)\right|=\log \left|\boldsymbol{\Theta}\left(\gamma^{*}\right)\right| .
$$

Therefore, we must have $\log \left|\Theta^{\prime}\left(\gamma^{*}\right)\right|=\log \left|\Theta\left(\gamma^{*}\right)\right|$, which, together with the uniqueness of $\boldsymbol{\Theta}\left(\gamma^{*}\right)$, implies $\boldsymbol{\Theta}^{\prime}\left(\gamma^{*}\right)=\boldsymbol{\Theta}\left(\gamma^{*}\right)$. This leads to a contradiction.

\section{REFERENCES}

[1] C. E. Shannon, "A mathematical theory of communication," Bell Syst. Tech. J., vol. 27, no. 3, pp. 379-423 and 623-656, Jul./Oct. 1948.

[2] U. Mittal and N. Phamdo, "Hybrid digital-analog (HDA) joint sourcechannel codes for broadcasting and robust communications," IEEE Trans. Inf. Theory, vol. 50, no. 5, pp. 1082-1102, May 2002.

[3] V. M. Prabhakaran, R. Puri, and K. Ramchandran, "Hybrid digital-analog codes for source-channel broadcast of Gaussian sources over Gaussian channels," IEEE Trans. Inf. Theory, vol. 57, no. 7, pp. 4573-4588, Jul. 2011.

[4] H. Behroozi, F. Alajaji, and T. Linder, "On the performance of hybrid digital-analog coding for broadcasting correlated Gaussian sources," IEEE Trans. Commun., vol. 59, no. 12, pp. 3335-3342, Dec. 2011. 
[5] A. Khina, Y. Kochman, and U. Erez, "Joint unitary triangularization for MIMO networks," IEEE Trans. Signal Process., vol. 60, no. 1, pp. 326-336, Jan. 2012

[6] A. Lapidoth and S. Tinguely, "Sending a bivariate Gaussian over a Gaussian MAC," IEEE Trans. Inf. Theory, vol. 56, no. 6, pp. 2714-2752, Jun. 2010.

[7] S. I. Bross, A. Lapidoth, and S. Tinguely, "Broadcasting correlated Gaussians," IEEE Trans. Inf. Theory, vol. 56, no. 7, pp. 3057-3068, Jul. 2010.

[8] R. Soundararajan and S. Vishwanath, "Hybrid coding for Gaussian broadcast channels with Gaussian sources," in Proc. IEEE Int. Symp. Inf. Theory (ISIT), Jun./Jul. 2009, pp. 2790-2794.

[9] Y. Gao and E. Tuncel, "Separate source-channel coding for transmitting correlated Gaussian sources over degraded broadcast channels," IEEE Trans. Inf. Theory, vol. 59, no. 6, pp. 3619-3634, Jun. 2013.

[10] C. Tian, S. Diggavi, and S. Shamai (Shitz), "The achievable distortion region of sending a bivariate Gaussian source on the Gaussian broadcast channel," IEEE Trans. Inf. Theory, vol. 57, no. 10, pp. 6419-6427, Oct. 2011.

[11] T. Kailath, A. H. Sayed, and B. Hassibi, Linear Estimation. Upper Saddle River, NJ, USA: Prentice-Hall, 2000.

[12] Z. Reznic, M. Feder, and R. Zamir, "Distortion bounds for broadcasting with bandwidth expansion," IEEE Trans. Inf. Theory, vol. 52, no. 8, pp. 3778-3788, Aug. 2006.

[13] K. Khezeli and J. Chen, "A source-channel separation theorem with application to the source broadcast problem," in Proc. IEEE Int. Symp. Inf. Theory (ISIT), Honolulu, HI, USA, Jun./Jul. 2014, pp. 2132-2136.

[14] L. Ozarow, "On a source-coding problem with two channels and three receivers," Bell Syst. Tech. J., vol. 59, no. 10, pp. 1909-1921, Dec. 1980.

[15] H. Wang and P. Viswanath, "Vector Gaussian multiple description with individual and central receivers," IEEE Trans. Inf. Theory, vol. 53, no. 6, pp. 2133-2153, Jun. 2007.

[16] J. Chen, "Rate region of Gaussian multiple description coding with individual and central distortion constraints," IEEE Trans. Inf. Theory, vol. 55, no. 9, pp. 3991-4005, Sep. 2009.

[17] L. Song, S. Shao, and J. Chen, "A lower bound on the sum rate of multiple description coding with symmetric distortion constraints," IEEE Trans. Inf. Theory, vol. 60, no. 12, pp. 7547-7567, Dec. 2014.

[18] R. Puri, K. Ramchandran, and S. S. Pradhan, "On seamless digital upgrade of analog transmission systems using coding with side information," in Proc. 40th Annu. Allerton Conf. Commun., Control, Comput. (Allerton), Monticello, IL, USA, Oct. 2002.

[19] V. M. Prabhakaran, R. Puri, and K. Ramchandran, "Colored Gaussian source-channel broadcast for heterogeneous (analog/digital) receivers," IEEE Trans. Inf. Theory, vol. 54, no. 4, pp. 1807-1814, Apr. 2008.

[20] A. D. Wyner and J. Ziv, "The rate-distortion function for source coding with side information at the decoder," IEEE Trans. Inf. Theory, vol. 22, no. 1, pp. 1-10, Jan. 1976

[21] M. H. M. Costa, "Writing on dirty paper (Corresp.)," IEEE Trans. Inf. Theory, vol. 29, no. 3, pp. 439-441, May 1983.

[22] D. P. Bertsekas, Nonlinear Programming, 2nd ed. Belmont, MA, USA: Athena Scientific, 1999
Lin Song received the B.Sc. degree in Electronic Information Engineering, the M.S. degree in Communication and Information Systems from the Harbin Institute of Technology, Harbin, Heilongjiang, China, in 2006 and 2008, respectively, and the Ph.D. degree in Electrical and Computer Engineering from McMaster University, Hamilton, ON, Canada, in 2012. She was a postdoctoral research assistant at McMaster University, Hamilton, ON, Canada from September 2012 to August 2013. She is currently with the Institute of Network Coding at the Chinese University of Hong Kong, Hong Kong, as a postdoctoral fellow. Her research interests include network information theory and coding theory.

Jun Chen (S'03-M'06) received the B.E. degree with honors in communication engineering from Shanghai Jiao Tong University, Shanghai, China, in 2001 and the M.S. and Ph.D. degrees in electrical and computer engineering from Cornell University, Ithaca, NY, in 2004 and 2006, respectively. He was a Postdoctoral Research Associate in the Coordinated Science Laboratory at the University of Illinois at Urbana-Champaign, Urbana, IL, from September 2005 to July 2006, and a Postdoctoral Fellow at the IBM Thomas J. Watson Research Center, Yorktown Heights, NY, from July 2006 to August 2007. Since September 2007 he has been with the Department of Electrical and Computer Engineering at McMaster University, Hamilton, ON, Canada, where he is currently an Associate Professor. His research interests include information theory, wireless communications, and signal processing. He received several awards for his research, including the Josef Raviv Memorial Postdoctoral Fellowship in 2006, the Early Researcher Award from the Province of Ontario in 2010, and the IBM Faculty Award in 2010. $\mathrm{He}$ is currently serving as an Associate Editor for Shannon Theory for the IEEE TRANSACTIONS ON INFORMATION THEORY.

Chao Tian (S'00-M'05-SM'12) received the B.E. degree in Electronic Engineering from Tsinghua University, Beijing, China, in 2000 and the M.S. and Ph.D. degrees in Electrical and Computer Engineering from Cornell University, Ithaca, NY in 2003 and 2005, respectively. Dr. Tian was a postdoctoral researcher at Ecole Polytechnique Federale de Lausanne (EPFL) from 2005 to 2007, and then was a member of technical staff-research at AT\&T Labs-Research in New Jersey. He joined the Department of Electrical Engineering and Computer Science at the University of Tennessee Knoxville in 2014, where he is now an Associate Professor. He was an Associate Editor for the IEEE SignAl Processing LetTERS from 2012 to 2014. His research interests include data storage systems, multiuser information theory, joint source-channel coding, quantization design and analysis, as well as image/video coding and processing. Dr. Tian received the Liu Memoria Award at Cornell University in 2004, and the AT\&T Key Contributor Award in 2010, 2011 and 2013 . 